\title{
Composing Synchronization and Real-Time Constraints
}

\author{
Lodewijk Bergmans and Mehmet Akşit ${ }^{1}$ \\ TRESE Project, Department of Computer Science, University of Twente, P.O. Box 217, 7500 AE Enschede, The Netherlands
}

\begin{abstract}
There have been a number of publications illustrating the successes of object-oriented techniques in creating highly reusable software systems. Several concurrent languages have been proposed for specifying reusable synchronization specifications. Recently, a number of real-time object-oriented languages have been introduced for building object-oriented programs with real-time behavior. Composing and reusing object-oriented programs with both synchronization and real-time constraints has not been addressed adequately, although most real-time systems are concurrent. This paper analyzes the origins of the problems in composing and reusing synchronization and realtime specifications, first as separate concerns, and later as composed behavior. To overcome the so-called inheritance anomaly problems, this paper proposes modular and composable synchronization and real-time specification extensions to the object-oriented model. The applicability of the proposed mechanisms is illustrated through a number of examples. ๑ 1996 Academic Press, Inc.
\end{abstract}

\section{INTRODUCTION}

Object-oriented analysis and design methods [50, 28] and programming languages such as $\mathrm{C}++$ [51] and Smalltalk [26] are now being applied to a large category of applications. Recently, a number of reusable objectoriented design patterns [24] have been introduced as a catalog of reusable object-oriented design knowledge. There have been a number of publications illustrating the successes of object-oriented techniques in creating highly reusable systems [45, 46]. These publications, however, largely deal with sequential systems without any consideration for concurrency and real-time aspects, although most real-world systems are concurrent and have some realtime aspect.

For several years, there have been many claims about the suitability of the object-oriented model for modeling concurrent systems. However, it appears that extensibility and reusability of concurrent applications is far from trivial. The problem that arises, the so-called synchronization constraint inheritance anomaly, has been extensively discussed in the literature $[35,39,15]$. The term inheritance anomaly means that introducing a new method and/or overriding an inherited method in a subclass may require additional

\footnotetext{
${ }^{1}$ E-mail: $\{$ bergmans | aksit\}@cs.utwente.nl.
}

redefinitions. Ideally, this should not be necessary. Several concurrent object-oriented languages $[31,22,38]$ have been introduced to solve the synchronization constraint inheritance anomaly problem, although most of these languages (still) suffer from this problem in one way or another.

Recently, there have been some attempts in defining real-time object-oriented languages [52, 17, 32, 27, 44]. These languages aim at reducing the complexity of applications through modularization so that predictability and reliability of applications can be increased. In addition, the inheritance mechanism can be useful in reusing welldefined and verified real-time programs. Similar to concurrent object-oriented languages, real-time object-oriented languages may suffer from the real-time constraints inheritance anomaly. In contrast to concurrent object-oriented languages, however, there has been almost no study on the origins of the real-time constraint inheritance anomaly problem. Needless to say, the combined analysis of concurrent and real-time constraint inheritance anomalies has not been addressed, although most real-time systems are concurrent.

It is important to note that both the synchronization and real-time constraint inheritance anomalies are not inherent in combining synchronization and real-time specifications with inheritance. On the contrary, the anomalies are largely language-dependent. How a language implements synchronization and real-time constraints and inheritance can be the major cause of inheritance anomalies.

This paper has two contributions. First, it presents generic object-oriented synchronization and real-time models which are useful in analyzing and relating synchronization and real-time constraint inheritance anomalies in a uniform way. These generic models help in searching for solutions that can deal with both problems. Based on these generic models, a number of important synchronization and real-time inheritance anomalies are identified and discussed.

Second, as a possible solution to both synchronization and real-time constraint inheritance anomalies, this paper proposes modular and composable synchronization and real-time specification extensions to the object-oriented model using the concept of composition-filters. Composition-filters can affect the synchronization and real-time characteristics of the received and sent messages. By proper configuration of the filters, one can specify synchronization and real-time constraints, as well as reuse of these 


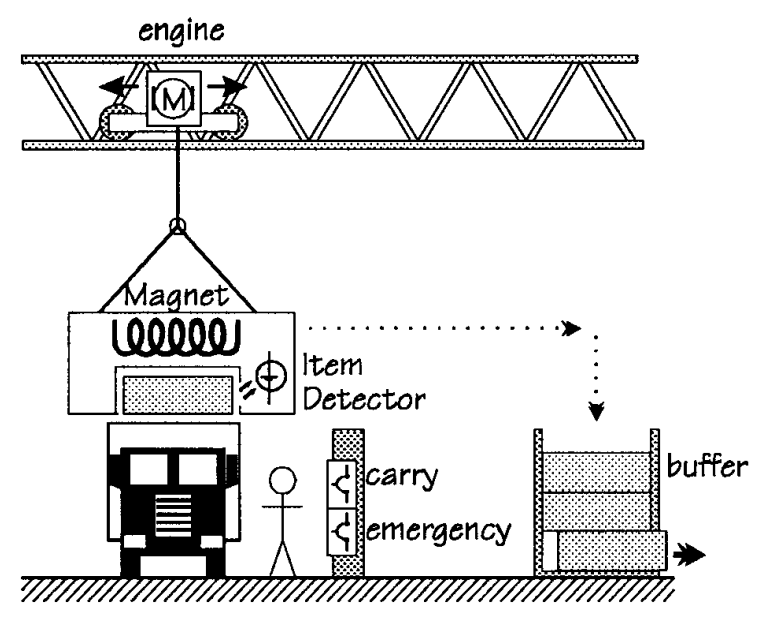

FIG. 1. A schematic overview of the crane system and its sensors.

constraints without causing inheritance anomalies. The applicability of the proposed mechanisms is illustrated through a number of examples.

The following section presents the example problem which is referred to throughout this paper. Sections 3 and 4 define the issues in composing synchronization and realtime constraints, respectively. Section 5 makes a combined analysis of the synchronization and real-time constraint composition problems. In addition, this section presents a number of requirements for successfully composing synchronization and real-time specifications together. Section 6 introduces the application of the composition-filters concept in solving the problems presented in this paper. The related work is presented in Section 7. Finally, Section 8 evaluates our approach and gives conclusions.

\section{AN EXAMPLE: A SOFTWARE CONTROLLED CRANE SYSTEM}

We present a simple example to explain the issues of composing synchronization and real-time constraints in object-oriented programs. Our example is a simplified model of a software controlled crane that can lift and carry containers from arriving trucks to a buffer area. The containers are taken from this buffer area for further handling. To carry the containers, the crane uses a magnetic latching mechanism. Figure 1 depicts this system.

The software is organized around an inheritance hierarchy which models different specializations of cranes. We will use an object-oriented pseudo-language for describing the examples; the meaning of the code examples should be self-explanatory.

Class Crane provides the basic functionality of the crane system, such as loading and carrying containers. In addition, class Crane stores and retrieves containers and inherits these buffering operations from class BoundedBuffer. In the following sections, we will describe classes BoundedBuffer and Crane.

\subsection{Definition of Class BoundedBuffer}

Class BoundedBuffer implements a generic storage mechanism with limited capacity. It offers a simple protocol consisting of the message get, which retrieves the earliest stored element from the buffer, and the message put (anElement) which adds the argument anElement to the buffer. In our crane example, the message put is used to add a container to the buffer area, and the message get is to be invoked when retrieving a container from this area. Figures 2 and 3 show the definition of the interface of class BoundedBuffer and the effect of the put and get messages on the crane system. Here, the methods Empty, Partial, and Full are defined for reading the state of the bounded buffer object.

The precise nature of the elements that are stored in a bounded buffer object is not relevant to this paper. In the definitions of methods put and get type Any is declared, which does not impose any type restrictions. The synchronization constraints of class BoundedBuffer will be discussed in Section 3.

\subsection{Definition of Class Crane}

Typical operation of the crane consists of a fixed sequence of actions for picking up a container from a truck and putting it in the buffer area. We express this sequence by the following series of messages to be sent to a crane object, for example, as the result of pressing the carry button depicted in Fig. 1:

crane.on;

latch the container by activating the magnetic field

crane.forward; move the crane and container to the buffer area

crane.put;

crane.off;

offer the container to the buffer area

release the container by turning off the magnetic field

crane.backward; move the crane backward to the truck

Here, the method put is inherited from class BoundedBuffer. The definition of the interface of class Crane is shown in Fig. 4. Note that a number of query methods have been

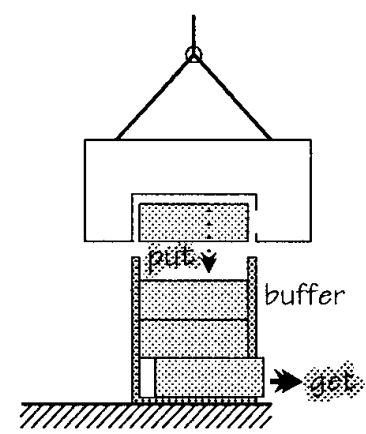

FIG. 2. The buffering of containers in the crane system. 


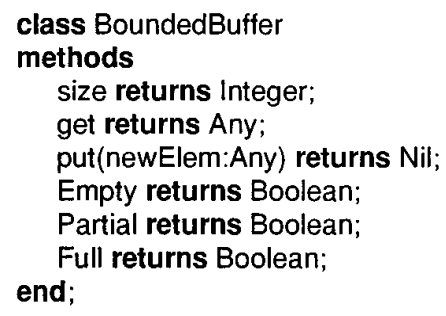

FIG. 3. Pseudo-code definition of class BoundedBuffer.

defined for reading the state of the crane object. For brevity, we have omitted method implementations.

\section{ISSUES IN COMPOSING SYNCHRONIZATION CONSTRAINTS}

This section studies the issues in composing synchronization constraints in concurrent object-oriented programs. First, the synchronization constraints of the crane system are specified. Second, a generic model is presented to analyze object-oriented synchronization composition mechanisms. Finally, possible synchronization inheritance anomalies are identified using the generic model.

\subsection{Synchronization Constraints of the Crane System}

To function properly, classes BoundedBuffer and Crane have to enforce certain synchronization constraints. Most object-oriented synchronization models define synchronization constraints using the states of objects and synchronization takes place only at the interfaces of objects prior to execution of methods. Referring to this model, we will describe in which state which messages should be blocked; in all other states the messages may proceed to execute.

\subsubsection{Synchronization Constraints of Class BoundedBuffer}

To avoid inconsistencies due to attempts of retrieving elements from an empty buffer or storing elements in a buffer that is already full, class BoundedBuffer must impose two synchronization constraints:

\section{(S1) if Empty then block get;}

This constraint specifies that if the buffer is empty (size $=$ 0 ), a received get message must be blocked until the buffer is no longer empty, which requires one or more invocations of put messages.

\section{(S2) if Full then block put;}

The constraint (S2) states that if the buffer is filled to its maximum capacity ( size $=$ limit), a received put message must be blocked until space becomes available. The latter requires the execution of one or more get messages.

\subsubsection{Synchronization Constraints of Class Crane}

This class introduces the following synchronization constraint (S3): The magnetic field may not be turned on as long as no container is in place to be latched.

(S3) if Unloaded then block on;

The method Unloaded returns a true value if there is no container in place (as signaled by the item detector in figure $1)$. Note that, as soon as a container is put in place, a previously received and blocked on method will be activated.

The synchronization constraint of a class is the composition of the synchronization specifications of that class and all its superclasses. For class Crane, this means that its synchronization constraint is the composition of (S1), (S2), and (S3).

\subsubsection{Extending Class Crane with Class ProtectedCrane}

For illustration purposes, we now introduce class ProtectedCrane which inherits from class Crane and introduces the synchronization constraint (S4):

(S4) if Moving then block on, off;

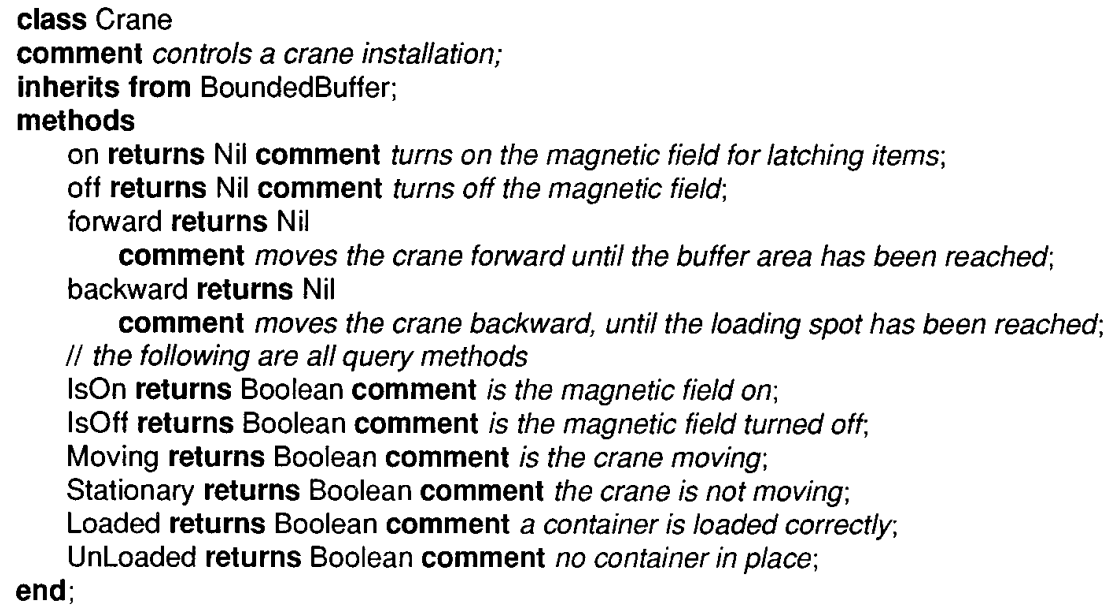

FIG. 4. Pseudo-code definition of class Crane. 


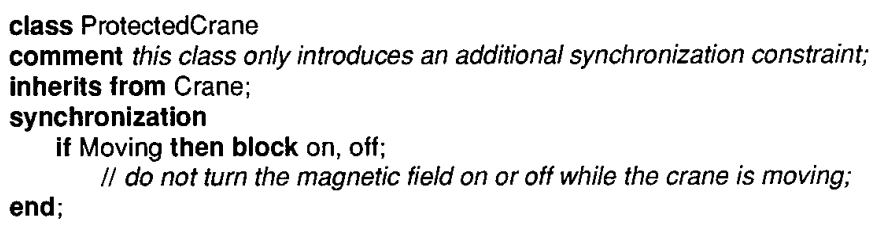

FIG. 5. Pseudo-code definition of class ProtectedCrane.

For safety purposes, the magnetic field is normally not allowed to be turned on or off while the crane is moving. The definition of the interface of class ProtectedCrane is shown in Fig. 5. Here, the synchronization constraint is specified in the synchronization clause.

\subsection{A Generic Model for Analyzing Synchronization Constraints}

To identify the potential problems in inheriting concurrent code, in this subsection we make an attempt at defining a generic object-oriented synchronization model. Our starting point is the assumption of the conceptual object model depicted in Fig. 6. In this model, each object has an object manager, a message queue, a collection of methods, and an object state. The object manager is responsible for scheduling: based on the state of the object, the state of the message queue and the synchronization constraints, it decides whether a received message can result in immediate method execution, or whether it will be put in the message queue. The object manager also takes care that queued messages will eventually lead to the execution of a method, once their synchronization constraints are satisfied. The object manager operates on one message at a time to ensure that competing messages are handled consistently. One of the most significant properties of this model is that synchronization is performed after the messages arrive at the object, and just before the execution of the corresponding methods.

One can identify a large number of factors thatpossibly-determine whether a message is acceptable or

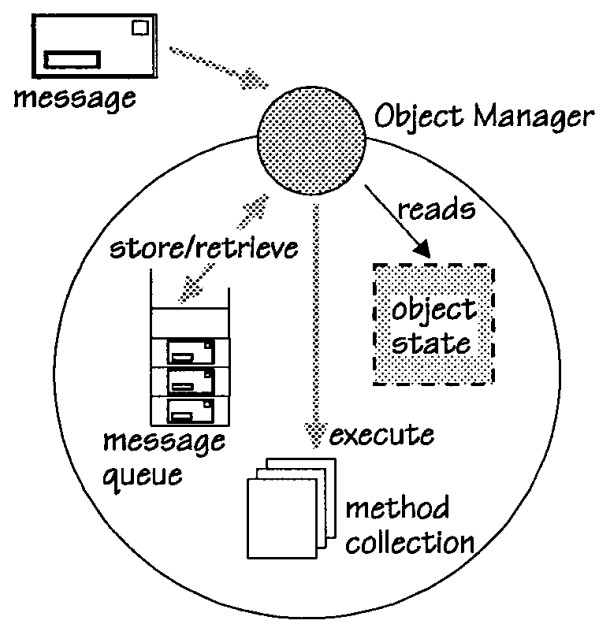

FIG. 6. A simplified model of the synchronization of messages. must be blocked. As stated before, message acceptance ultimately depends on the state of the object, in the broadest sense possible. We designate this by the term implicit object state to avoid confusion with the common use of "object state," which indicates the values of instance variables only. The implicit object state may include the values of instance variables, the properties of the message such as the message selector ${ }^{2}$ and the message arguments, the current activities within the object (for example, the number of active threads), the message queue (i.e., what other messages are waiting to be served), and the history of the object (for instance, which messages have been executed previously and how often). A particular concurrent objectoriented language may express one or more of these, or may even offer other possible state information.

The implicit object state is affected by several types of events: the arrival of new messages, the acceptance of a message or start of a message execution, the termination of a method execution, and the effects of method executions themselves. The implicit object state can be described as a region in a large state space. To make the implicit object state explicit in a program, state abstraction is required, resulting in a number of (synchronization) conditions. Each condition provides a concrete specification of the implicit state of the object.

For example, for a bounded buffer object, we can define the following three synchronization conditions:

$$
\begin{array}{ll}
\text { Empty: } & \text { self.size }=0 \\
\text { Partial: } & (\text { self.size }>0) \text { and }(\text { self.size }<\text { limit }) \\
\text { Full: } & \text { self.size }=\text { limit }
\end{array}
$$

Conditions have two important properties. First, they perform extraction of the relevant state information, and second, they create an independence between the implementation-dependent implicit object state and the message-related synchronization code.

Synchronization of messages is modeled as a set of acceptable (nonblocked) messages, the so-called accept set. Synchronization can be specified by defining the mapping between the synchronization conditions and the set of possible accept sets of an object. The message accept set is offered to the scheduler, which uses it to activate-or postpone-the execution of messages. Figure 7 shows how the elements of the model are related.

A synchronization specification according to this model thus requires the following components:

1. State Abstraction. Maps the implicit object state to synchronization conditions.

2. Synchronization Condition Mapping. Maps the synchronization conditions to message acceptance.

Consider, for example, the language ACT ++ [31]. In $\mathrm{ACT}++$, synchronization is specified through so-called behavioral abstraction. For this purpose, every object de-

\footnotetext{
${ }^{2}$ Message selector is a term used within the object-oriented community. It denotes the method to be invoked once the message is accepted.
} 


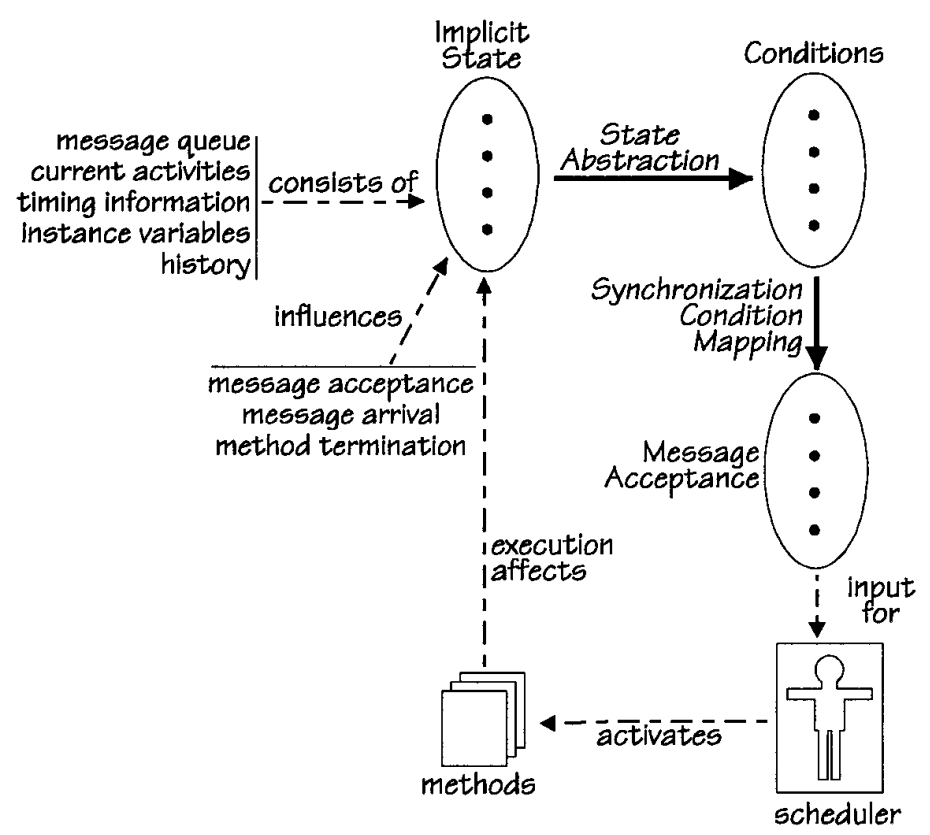

FIG. 7. Schematic outline of synchronization specifications.

fines a set of behavior names and for every behavior name the methods that are accepted for that behavior are listed. Every method specifies the next behavior with a become statement. The behavioral abstractions in ACT ++ correspond to the synchronization conditions in our generic model. The become statements implement the state abstraction, and the definition of acceptable methods for each behavior realizes the condition mapping.

In some synchronization schemes, the mapping from the implicit object state to method acceptance is made immediately, without the intermediate step of synchronization conditions. This is the case in guard-like approaches, for example, in the Guide language [20]. A method guard directly maps the current state of the object to message acceptance.

Most concurrent object-oriented languages that support interface control and object-level synchronization can be described by this model. We therefore use this generic model to describe the problems with reuse of synchronization code in a largely language-independent way.

\subsection{Synchronization Constraints Inheritance Anomalies}

To avoid problems in reusing synchronization constraints through inheritance, the following requirements must be satisfied:

1. Modularity of synchronization specifications.

2. Composability of synchronization constraints.

3. Expressiveness for state abstractions.

The origins of inheritance anomalies can be traced back to ignoring one or more of these requirements, which are discussed in the following subsections.

\subsubsection{Modularity of Synchronization Specifications}

The modularity of synchronization specifications means that the synchronization specification is separated from the application code of the class. In fact, not only the synchronization-related specifications must be completely separated from the application code, but the synchronization specification must consist of an independent State Abstraction and Condition Mapping Functions, as depicted in Fig. 7. The prime reason for requiring synchronization modularity is to reduce the dependencies between the application code,such as method implementations, and the acceptance of messages. Because in subclasses the synchronization will virtually always change (if only to cope with newly added methods), dependencies between application code and message acceptance will require the redefinition of application code in many situations.

\subsubsection{Composability of Synchronization Constraints}

The composability of synchronization constraints is an important factor for effective reuse and extension of synchronization constraints. The synchronization specification of a class must not be a monolithic unit. Specialization, extension or reuse of a monolithic synchronization specification in subclasses is not possible as it is not possible to replace parts of the specification. Adding new constraints to the specification is only feasible in specific cases (e.g., when the additional constraints are fully orthogonal to the existing specification).

If we consider the composability problem in our generic synchronization model, we must focus on Synchronization Conditions and on Synchronization Condition Mapping. Synchronization Conditions provide for an intermediate 
that is independent of both the implementation details of the object and the precise interface specification. They function as a reusable "abstract synchronization specification" that does not violate encapsulation and can be tailored to the exact interface of the reusing client class. ${ }^{3}$ This is because the synchronization conditions conform to logical states of the object with associated consistency constraints, such as UnLoaded, Loaded, Stationary, and Moving for class ProtectedCrane.

For optimum composability, we require that every synchronization condition can be associated with the acceptance of multiple methods and that synchronization condition mappings can be synthesized into a single new condition mapping. We term the first requirement as polymorphic synchronization specifications and the second one as synchronization synthesis. These two requirements are discussed now.

Polymorphic Synchronization Specifications. The requirement for polymorphic synchronization specifications is based on the goal of reusing and extending synchronization specifications. This requires that a certain (synchronization) constraint must be applicable to multiple methods, either in the same class or in subclasses.

Synchronization Synthesis. The term synthesis applies to the combination of multiple predefined components into a new one as well as the extension of a component with new, local, components. Synchronization synthesis is essential for the reuse and extension of synchronization constraints.

Consider, for example, the synchronization constraints of classes Crane and ProtectedCrane. Class Crane restricts the applicability of the method on; if there is no container in place, then the method on must be delayed. Class ProtectedCrane extends this restriction further. To execute the method on, the crane must be in a stationary state. These two constraints remain both valid in class ProtectedCrane, so what is needed is a synthesis of synchronization constraints. The problem here is that we need to chose the semantics for synthesizing the synchronization constraints. The most obvious is an AND semantics: only if both constraints are satisfies will the corresponding method be activated. For certain problems, oR semantics may be required. This is discussed in Section 8.1.4. Synthesizing synchronization constraints is even more difficult when combining two concurrent classes through multiple inheritance. We refer to [13] for more details on this topic.

In our generic synchronization model, the property of composable synchronization specifications is a characteristic of Synchronization Condition Mapping. In particular, with each synchronization condition multiple acceptable messages must be associated, and it must be possible to extend the mapping incrementally, in order to associate

\footnotetext{
${ }^{3}$ Note that synchronization conditions do not imply a particular synchronization scheme; they can range from behavioral abstractions to guards.
}

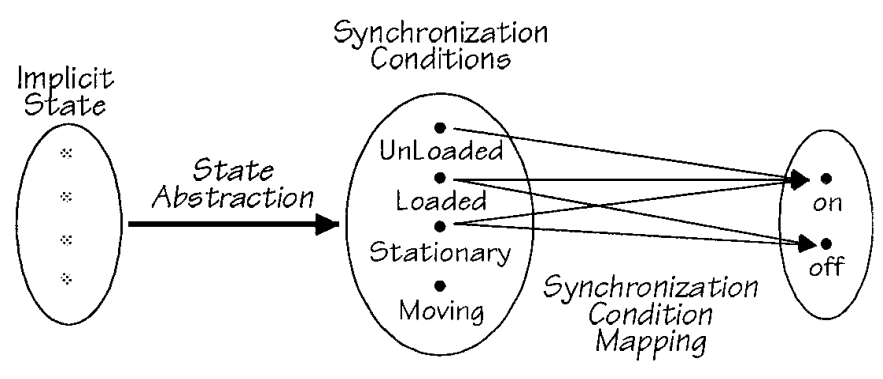

FIG. 8. Example of composability in the generic model.

additional messages with an existing synchronization condition (as exemplified by the union operation on enabled sets in [53]). In Fig. 8, the condition mapping is shown for the constraints specified in classes Crane and ProtectedCrane.

\subsubsection{Expressiveness for State Abstractions}

Insufficient expressiveness for synchronization conditions can reveal itself in two-closely related-ways: it may completely prohibit the expression of specific synchronization constraints. However, it may also be possible to "program around" the problem, by writing additional application code (i.e., in methods). The latter situation is a frequent source for inheritance anomaly.

One particular example of this is the so-called historysensitiveness $[35,37]$, which is exemplified by an extension to the bounded buffer. Suppose that we want to add a subclass of BoundedBuffer that provides the method gget. This is an ordinary get operation, except that it should not execute immediately after a put. The problem with this example is that the state abstraction specification must be able to express the history (of received messages) of the object. This can be provided by the system (cf. the wait once transitions in [38]), or the application must do some explicit bookkeeping.

In the gget example, assume that there is no special language support for keeping a history administration. Then the only way to know-inside a synchronization specification-that the latest executed method was a put, is by modifying all the methods of the class to maintain a Boolean status variable, say justPut.

Note that we can use the implementations of the superclass methods by using, for example, the pseudo-variable super as defined in the Smalltalk language. However, this does not make the inheritance anomaly less severe. Not only must all methods be redefined (including the ones inherited indirectly), but in the subclasses the justPut Boolean must be maintained as well by all methods!

The relevance of this example is to demonstrate how lack of expressiveness can cause inheritance anomalies. This can be avoided through the ability to make a mapping from any implicit object state to a synchronization condition. The problem, however, is that the notion of implicit object state is quite broad and, in fact, system-dependent. 


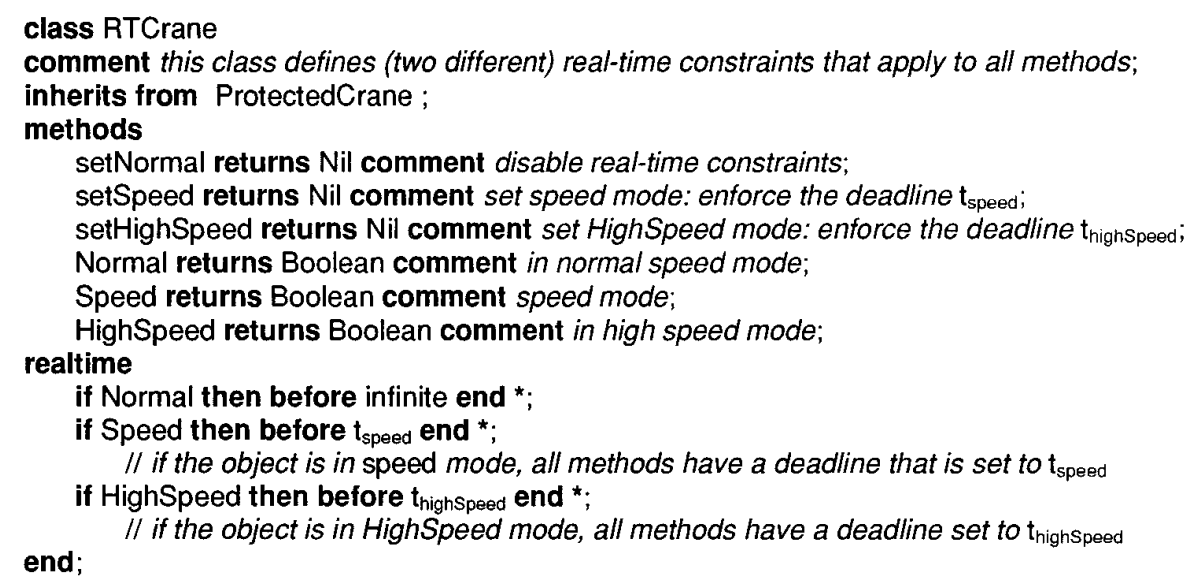

FIG. 9. Pseudo-code definition of class RTCrane.

\section{ISSUES IN COMPOSING REAL-TIME CONSTRAINTS}

In this section, first the real-time constraints of the crane system are defined. Second, a generic model is introduced to analyze object-oriented real-time specification techniques. Finally, with the help of this generic model realtime inheritance anomalies are identified.

\subsection{Real-Time Constraints in the Crane System}

We will now continue extending the inheritance hierarchy of the crane system by introducing new classes that specify real-time constraints. For brevity, we only show deadlines on method invocations and neglect other realtime specifications such as specifying starting times and periodicity. ${ }^{4}$

\subsubsection{Definition of Class RTCrane}

Class RTCrane is an extension of class ProtectedCrane and has three modes: Normal, Speed, and HighSpeed. If an instance of class RTCrane is in Normal mode, then no real-time constraints are enforced. If the instance is in Speed mode, then all its interface methods (both local and inherited) have to be completed in $t_{\text {Speed }}$ time units. If the instance is in HighSpeed mode, then all the interface methods have to be completed in $t_{\text {HighSpeed }}$ time units, where $t_{\text {HighSpeed }}<t_{\text {Speed }}$. The instances of class RTCrane can be put into various modes to cope with the changing demands of its operating environment. Increasing the speed of a crane increases the energy consumption and restricts the maximum allowable weight of the containers to be carried. The modes of an instance can be changed dynamically. The definition of class RTCrane is shown in Fig. 9.

The mode of the object can be changed to Normal, Speed, and HighSpeed modes, by invoking the methods setNormal, setSpeed and setHighSpeed, respectively. The

\footnotetext{
${ }^{4}$ Specifying starting times and periodicity does not introduce additional problems in real-time compositions. The reader can refer to $[9,19]$ to obtain more information about real-time specification techniques.
}

real-time constraints are specified in the realtime clause. Depending on the condition specified between the if .. then clause, the construct before $\langle$ deadline $\rangle$ end $\langle\operatorname{method}(s)\rangle$ specifies that the execution of all $\langle\operatorname{method}(s)\rangle$ is to be terminated before the time unit $\langle$ deadline $\rangle$. The deadline infinite indicates no deadlines is imposed. A wildcard "*" is used to designate all the interface methods of the object including the inherited methods.

\subsubsection{Definition of Class EmergencyRTCrane}

As a final example, consider class EmergencyRTCrane which inherits from class RTCrane and introduces the method emergency. The method emergency immediately shuts off the magnetic field, no matter what the current state of the crane is. This method can be invoked as the result of pressing an emergency button. The interface of class EmergencyRTCrane is defined in Fig. 10.

\subsection{A Generic Model for Analyzing Real-Time Constraints}

In this subsection we make an attempt at defining a generic object-oriented real-time model. We assume that timing information travels together with the message. In this way, timing information imposed by the caller of a message is made available to the receiver, which may additionally modify the timing information. The application of timing constraints may depend on the actual state of the object (this includes the timing information of the current message). This is shown in Fig. 11.

The generic model for real-time constraints shares parts with the generic model for synchronization discussed in the previous section: It is based on Implicit State, which is mapped through state abstraction to Real-Time Conditions. Real-Time Conditions are the necessary conditions for applying the Real-Time Constraints.

The combination of Real-Time Condition Mapping and Real-Time Constraints results in Message Timing Constraints, which define-optional-timing constraints for 


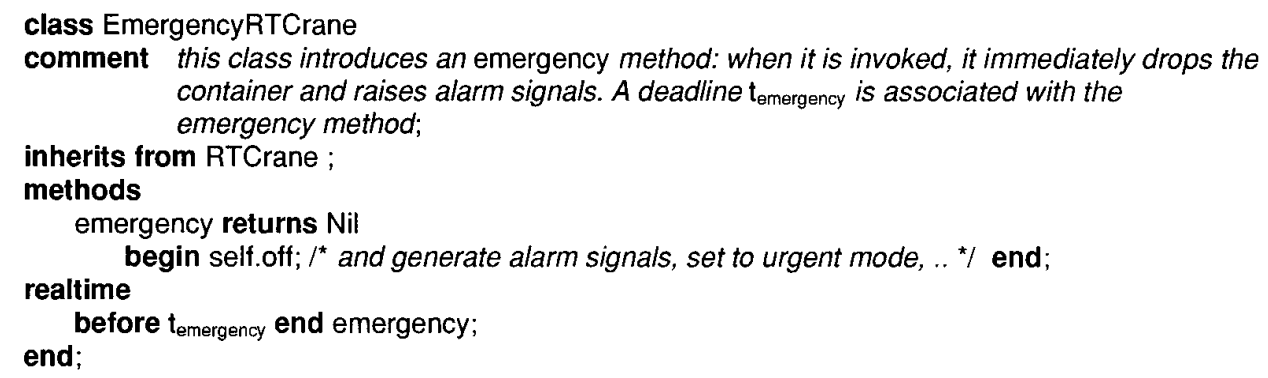

FIG. 10. Pseudo-code definition of class EmergencyRTCrane.

each message. The result of the message timing constraints and the available timing information of the message is offered to Scheduler, ${ }^{5}$ which uses these to schedule and activate method executions. The elements involved in the specification of real-time constraints are shown in Fig. 12.

A real-time constraint specification according to this model thus requires the following components:

1. State Abstraction. Maps the implicit object state to real-time conditions.

2. Real-Time Condition Mapping. Maps the real-time conditions to message timing constraints.

Real-Time Condition Mapping differs from Synchronization Condition Mapping through the fact that it includes timing specifications.

\subsection{Real-Time Specification Inheritance Anomalies}

The discussion of real-time constraint inheritance anomalies is very similar to the discussion of synchronization

\footnotetext{
${ }^{5}$ The concept of real-time scheduling can be considered in a broader sense; scheduling corresponds to any possible implementation of realtime constraint specifications, including mapping to parallel machines and choosing among alternative implementations.
}

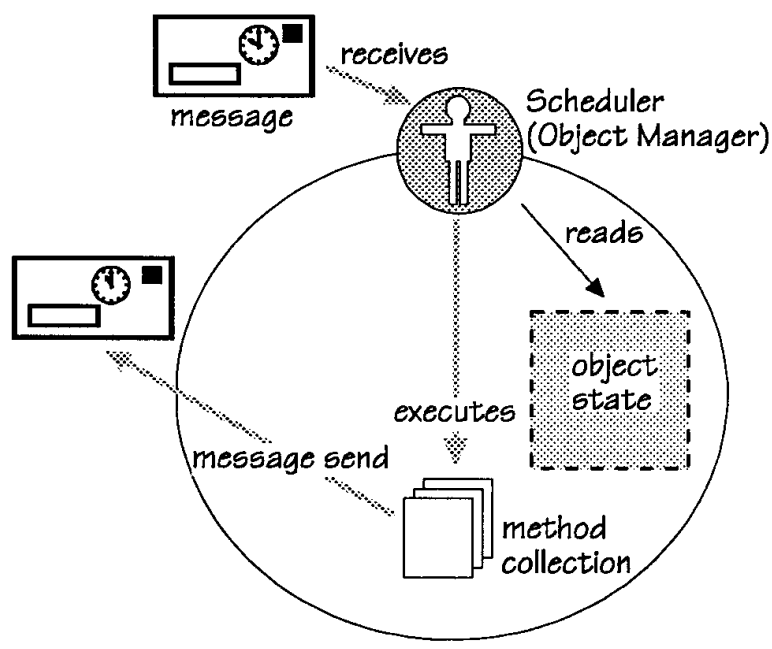

FIG. 11. A simplified model of the real-time scheduling of messages. constraint inheritance anomalies, only the components of the model differ somewhat.

Similar to the requirements given in Section 3.3, to avoid problems in reusing real-time constraints through inheritance, the following requirements must be satisfied:

1. Modularity of real-time specifications.

2. Composability of real-time constraints.

3. Expressiveness for state abstractions and timing specifications.

\subsubsection{Modularity of Real-Time Specifications}

The prime requirement to avoid inheritance anomalies is that the language provides modular specifications for State Abstraction and for Real-Time Constraint/Condition Mapping. If the real-time specifications cannot be separated from the method implementations, it is impossible to redefine the real-time specification nor the method implementations without redefining both. In addition, to avoid implementation dependencies, the implementation

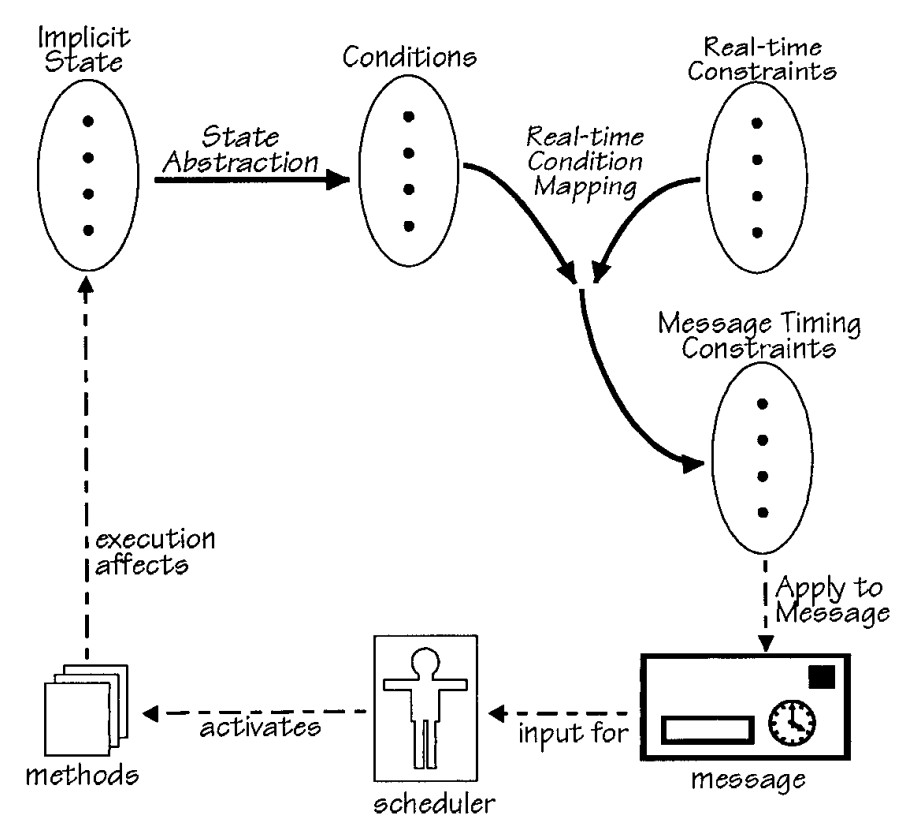

FIG. 12. A generic model for real-time constraints. 
of the choice of (or between) real-time constraints must be separated.

\subsubsection{Composability of Real-Time Constraints}

We will study the real-time composability problem in two parts: the polymorphism requirement and the synthesis requirement.

Polymorphic Real-Time Specifications. In Section 4.1.1, class RTCrane was defined with a number of realtime constraints that were applicable to all its methods. In general, real-time object-oriented languages do not provide such a specification construct, and therefore, the same real-time specification must be defined repeatedly. This causes two kinds of problems. First, the intuitive real-time specification states that the deadline is imposed on all methods of the class. This should be also true when additional methods are introduced in subclasses. This implies that all subclass implementors must be aware of this realtime constraint and add it to every newly defined method. Secondly, assume that a deadline is to be modified (either in the same class or in a subclass), then all the real-time constraint specifications must be updated.

Real-Time Synthesis. The combination of real-time constraints from separate classes, e.g., through (multiple) inheritance, may cause semantic interference, which will require additional redefinitions. As an example, assume that we modify class EmergencyRTCrane such that it imposes a certain deadline upon all methods of the class when the class is in the emergency state. In this case, there is a potential conflict with the real-time constraints defined in class RTCrane for the Speed and HighSpeed modes. This may require-depending on the specific real-time constraint specification scheme-redefinition of the realtime constraints.

\subsubsection{Expressiveness for State Abstractions and Timing Specifications}

The reason that lack of expressiveness for state abstractions and for timing constraints causes inheritance anomaly is the same as in the discussion for synchronization inheritance. For example, assume that an object's real-time con- straints depend on the history of the executions of some inherited methods. The problem here is similar to the one discussed in Section 3.3.3; the state abstraction specification must be able to express the history (of received messages) of the object.

\section{ISSUES IN COMPOSING SYNCHRONIZATION AND REAL-TIME CONSTRAINTS}

\subsection{Synchronization and Real-Time Compositions in the Crane System}

In Table I, we illustrate the inheritance hierarchy of the crane system including both the synchronization and realtime constraints in the form of a table. In the left-most column, the inheritance hierarchy is shown. Each classexcept class BoundedBuffer-inherits from the class in the row above it. For the proper functioning of all classes, the inheritance mechanism of the adopted language must be able to compose the synchronization and real-time specifications properly; all constraints must be satisfied without redundant redefinitions of methods and constraints.

Classes BoundedBuffer and Crane introduce synchronization constraints on different methods.

Classes Crane and ProtectedCrane both specifycomplementary-constraints for the same method on. This indicates that the inheritance mechanism must be able to compose synchronization constraints defined for the same method.

ProtectedCrane specifies the same synchronization specification for the methods on and off (S4). An extreme example of such a polymorphic synchronization constraint would be: "full: block *". For example, if the buffer area is full, then all operations of the crane could have been blocked by this synchronization constraint.

The real-time constraints of class RTCrane (R1) and (R2) may conflict with the synchronization constraints of the methods on, off, put, and get, which are defined by classes BoundedBuffer, Crane, and ProtectedCrane. If one or more of these methods are blocked due to synchronization constraints, then the real-time constraints as specified by (R1) and (R2) may not be satisfied.

Class EmergencyRTCrane defines a real-time constraint for the method emergency; if the method emergency is

TABLE I

The Crane Classes with Their Synchronization and Real-Time Constraints

\begin{tabular}{lll}
\hline \multicolumn{1}{c}{ Class hierarchy } & \multicolumn{1}{c}{ Synchronization constraint } & Real-time constraint \\
\hline BoundedBuffer & (S1) Empty: block get & - \\
Crane & (S2) Full: block put & \\
ProtectedCrane & (S3) Unloaded: block on & \\
RTCrane & (S4) Moving: block on, off & - \\
EmergencyRTCrane & Synchronization-real-time conflict & (R1) Speed: before $t_{\text {speed }}$ end $^{*}$ \\
& Synchronization-real-time conflict & (R2) HighSpeed: before $t_{\text {Highspeed }}$ end $^{*}$ \\
\hline
\end{tabular}


invoked, then the magnetic field must be turned off immediately. However, class ProtectedCrane delays off messages while the crane is moving. The real-time constraint of EmergencyRTCrane, therefore conflicts with the synchronization constraint of ProtectedCrane if the method emergency is invoked while the crane is moving.

The synchronization constraints are defined to preserve the logical behavior of objects. For example, if the magnetic field is turned off while the crane is moving, then the container may fall down and be damaged.

A real-time system is a system in which the correctness of its behavior depends not only on the logical computations carried out but also on the time the results are delivered. The built-in notion of time and how it is used in the system is the difference between real-time systems and non-realtime systems. The so-called hard timing constraints define a time-bound for a process that must be fulfilled, otherwise the computed result is useless, or can even be harmful. The process is not allowed to execute outside the specified time-bound. For example, in the crane system if the method emergency is invoked because the crane operator is in danger, then carrying the container must be stopped immediately. Soft timing constraints define a time-bound for a process outside which the computed result is not useless but still has a (diminished) value. The process is allowed to continue outside the specified time-bound.

\subsection{Requirements for Composing Synchronization and Real-Time Constraints}

From the discussion in the previous sections, we infer several requirements which object-oriented concurrent and real-time languages should fulfill, in order to be considered suitable for constructing extensible and reusable applications:

- Eliminating inheritance anomalies through separation of concerns. The adopted object-oriented language must enable the software engineer to define and reuse synchronization and real-time constraints separately or together without causing inheritance anomalies. In Section 3.2, Implicit State, Synchronization Conditions, Message Acceptance, Scheduler, and Methods are defined as the essential elements of the generic synchronization model. The mapping functions State Abstraction, Synchronization Condition Mapping, and Method Executions relate these elements to each other. The generic real-time model extends the synchronization model with Real-Time Condition Mapping and Real-Time Constraints. Some of these elements and functions are implicit, such as Implicit State, Scheduler, and Message Executions. The basic requirement for eliminating inheritance anomalies is that the adopted language must separate the synchronization and real-time elements and mapping functions from each other. In addition, the inheritance mechanism of the language must be able to compose these along the inheritance hierarchy.
- Expression power. The adopted language must provide sufficient expression power to deal with different synchronization and real-time requirements. For example, the State Abstraction Function must be expressive enough to represent various implicit states. Similarly, the Real-Time Condition Mapping and Real-Time Constraints must be able to specify different real-time requirements.

- Assign priorities to specifications. If synchronization and real-time specifications conflict with each other, then the software engineer must be able to assign priorities to synchronization and real-time constraints. While assigning priorities, the software engineer may consider two important cases:

1. Synchronization constraints are likely to prevail soft timing constraints. Defining priorities can be based on analyzing critical states. Relatively the most unwanted state must be avoided. Consider the conflicts between real-time and synchronization constraints of classes RTCrane and ProtectedCrane. If the method off is invoked on an instance of class RTCrane and this instance is in HighSpeed mode and is moving, then the synchronization constraint of ProtectedCrane will defer the request until the crane is at Stationary state. Giving higher priority to real-time constraint may increase the speed but will possibly result in damage to the container. In such a case, it is preferable that the synchronization constraint has a higher priority than the real-time constraint. Note that the real-time constraints of RTCrane are soft timing constraints.

2. Hard timing constraints are likely to prevail synchronization constraints. Consider, for example, the following situation. The method emergency is invoked because the crane operator is in danger and the further movement of the container would make the situation even worse. Turning off the magnetic field and possibly damaging the container is definitely preferable to risking the life of the operator. Note that the real-time constraints of EmergencyRTCrane are hard timing constraints.

\section{THE COMPOSITION FILTERS APPROACH}

In this section, first we will present an object-oriented model to express composition of synchronization and realtime constraints. This model is based on the compositionfilters concept. Second, we will model the crane system using this approach.

\subsection{Extending the Object-Oriented Model with Composition Filters}

In Section 5.2, separation of concerns was presented as one of the fundamental requirements in eliminating inheritance anomalies. The conventional object-oriented model as adopted by languages like $\mathrm{C}++$ and Smalltalk provides features that are very useful for a large category of applications and therefore it must be kept as a useful abstraction mechanism. However, to solve the identified 


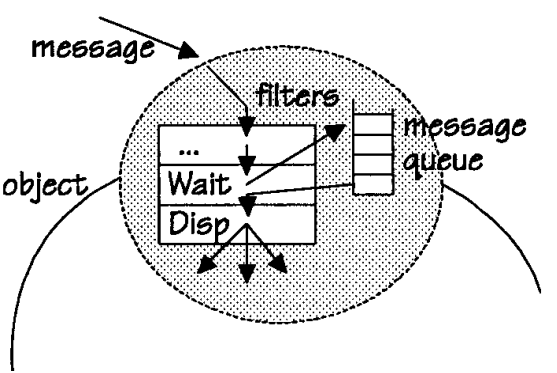

FIG. 13. The manipulation and buffering of messages at the filters of an object.

problems,${ }^{6}$ the object-oriented model must be enhanced modularly without losing its basic features. For this purpose, the composition-filters model extends the conventional model through a set of so-called filters. ${ }^{7}$

Each message that arrives at an object is subject to evaluation and manipulation by the filters of that object. In Fig. 13, the manipulation of messages by the filters of an object is depicted. Each filter deals with a particular concern. In composing synchronization and real-time constraints there are basically three concerns:

1. Composition of synchronization constraints, handled by Wait filters.

2. Composition of real-time constraints, handled by RealTime filters.

3. Inheriting methods and executing messages, handled by Dispatch filters.

\footnotetext{
${ }^{6}$ As well as other identified problems such as the obstacles defined in [6].

${ }^{7}$ The composition-filters approach is a modeling paradigm rather than the definition of a particular language with fixed semantics. Compositionfilters can be attached to objects expressed in different languages, such as Sina [2, 3], C++ [25], and Smalltalk [21]. The semantics of objects expressed in the composition-filters model can be largely determined by the semantics of the filters. Several different filter types have been defined in the past. For example, [2] illustrated how both inheritance and delegation can be simulated using filters. In [4], filters were introduced for defining reusable transactions. Language-database problems were addressed in [5]. In [7], filters were used to abstract coordinated behavior among objects. The application of composition-filters for real-time specifications was published in [8].
}

The aim of the composition-filters model is to improve the expression power of the conventional object-oriented model through modular and orthogonal extensions rather than building increasingly complex object structures. In the following sections, we will illustrate how compositionfilters can be applied to compose synchronization and realtime constraints without creating inheritance anomalies.

\subsection{Synchronization in the Composition-Filters Model}

In Fig. 14, we show the interface definition of class BoundedBuffer expressed using the Sina language. The major extensions to the conventional object-oriented model are condition and filter declarations, as highlighted by gray boxes. Class BoundedBuffer declares the conditions Empty, Partial and Full, which correspond to the three states of a bounded buffer. The second extension is the declaration of two filters following the inputfilters ${ }^{8}$ clause. A filter determines whether a particular message can be accepted or rejected. It also determines what action is to be performed in either case. Each filter is declared as an instance of a filter class. An arbitrary number of filters may be declared in a class definition. Class BoundedBuffer declares two filters, instances of class Wait and class Dispatch. This is because a bounded buffer object has two concerns: synchronization, handled by the wait filter and method execution, handled by the Dispatch filter.

A filter of class Wait has the following intuitive definition: When a message arrives at a wait filter, it can only proceed when the message is accepted by the filter, and it will be blocked otherwise, until the message can be accepted by the filter. In class BoundedBuffer, the input filter bufferSync of class Wait is declared as

$$
\begin{aligned}
& \text { bufferSync : Wait }=\{\text { Empty }=>\text { put, Partial }=>\text { put, get }\}, \\
& \text { Full =>get }\} \text {; }
\end{aligned}
$$

When a message is received by an instance of class BoundedBuffer, it is first applied to the wait filter. This

\footnotetext{
${ }^{8}$ In addition to input filters, the composition-filters model also supports output filters. Output filters affect outgoing messages, whereas input filters affect incoming messages. For brevity, we do not further consider output filters in this paper.
}

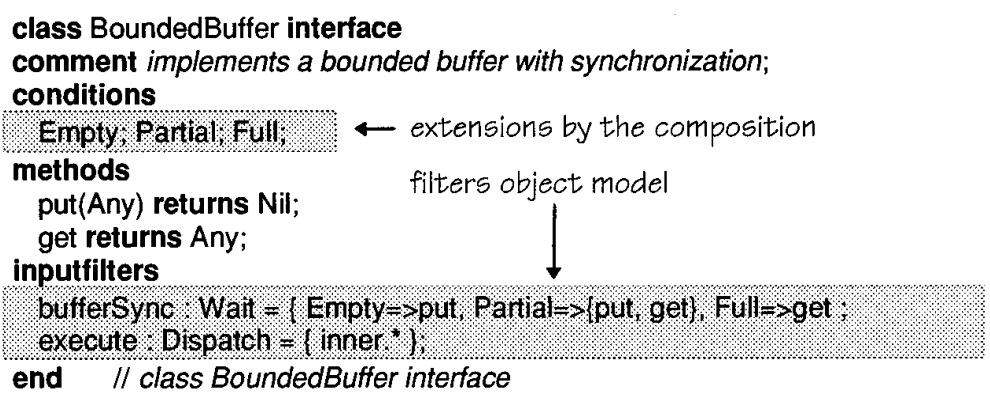

FIG. 14. Sina definition of the interface of class BoundedBuffer. 
wait filter has three parts separated by the operator ",". The first part "Empty=>put" has the following meaning: If the received message has the selector put and the condition Empty evaluates to true, then the message matches this part and will be forwarded to the dispatch filter. Otherwise, the message is evaluated by the second part. The second part matches the message if the message has the selector put or get, and if the condition Partial evaluates to true. If both parts do not match, then the received message is evaluated by the third part expressed as "Full = > get." This part matches the message if the condition Full evaluates to true and if the message has the selector get. If the received message does not match any of these parts, then it will be blocked until one of the parts will match the message.

The Dispatch filter defines a simple matching mechanism that maps all received messages to the local ("inner") methods. The wild card "*" implies that all message selectors - that are supported by the object (inner)-are acceptable. The Dispatch filter takes care that a message that does match will be dispatched. Dispatching to a local method results in execution of that method.

To illustrate the synchronization of messages, we show the message queue for an instance of class BoundedBuffer that subsequently receives the messages put, get, get', get",

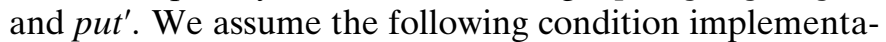
tions for class BoundedBuffer:

\section{conditions}

Empty begin return noOfElements $=0$ end; // no elements in the buffer

Partial begin return (Empty.not and Full.not) end; // not empty, not full

Full begin return noOfElements $=$ maxSize; end; $/ /$ the buffer is full

The subsequent states of the message queue of the object resulting from the arrival, buffering and dispatching of messages are shown in Fig. 15: As the first message put is received (no. 1), it is matched against the wait filter
bufferSync. The message put matches the first filter element, as the Empty condition is valid. Thus the message is immediately dispatched and therefore removed from the queue again (no. 2). Now the buffer contains one element, causing condition Partial to become true. So when the message get arrives (no. 3), it is accepted by the filter, and immediately dispatched (no. 4). Condition Partial is no longer valid, causing the received message get' to be blocked (no. 5). When the next message get" arrives, it is placed in the queue, after the message get' (no. 6).

When put' arrives, it will be placed at the tail of the queue (no. 7), but since it is the first acceptable message in the queue, it will be dispatched prior to the get messages (no. 8). After put' has completed its execution (as we assume mutual exclusion here, which is enforced by default), condition Partial is valid once again. This enables both get $^{\prime}$ and get $^{\prime \prime}$, in which case the first applicable message $\left(g^{\prime} t^{\prime}\right)$ in the queue is dispatched. After the execution of get', the condition Empty will become true and condition Partial will be false, therefore get" remains in the queue (no. 9).

\subsubsection{Definition of Class Crane Using Filters}

Now we will illustrate how class Crane can be implemented using the composition-filters approach. Consider the interface definition of class Crane in Fig. 16. The methods that allow reading the state of the object are declared following the conditions clause. Further, class Crane declares container of class BoundedBuffer as an internal object. Internal objects are used to implement compositionbased inheritance as will be explained in the following paragraphs.

Class Crane declares two filters: sync of class Wait and compose of class Dispatch. If a message is received by an instance of class Crane, then it will be first applied to the wait filter. This filter has two parts. The filter part "Loaded $=>$ on" means that the message with the selector on can match this part only if the condition Loaded evaluates to true. If the message cannot match this part, then it will be evaluated with respect to the next part. The second

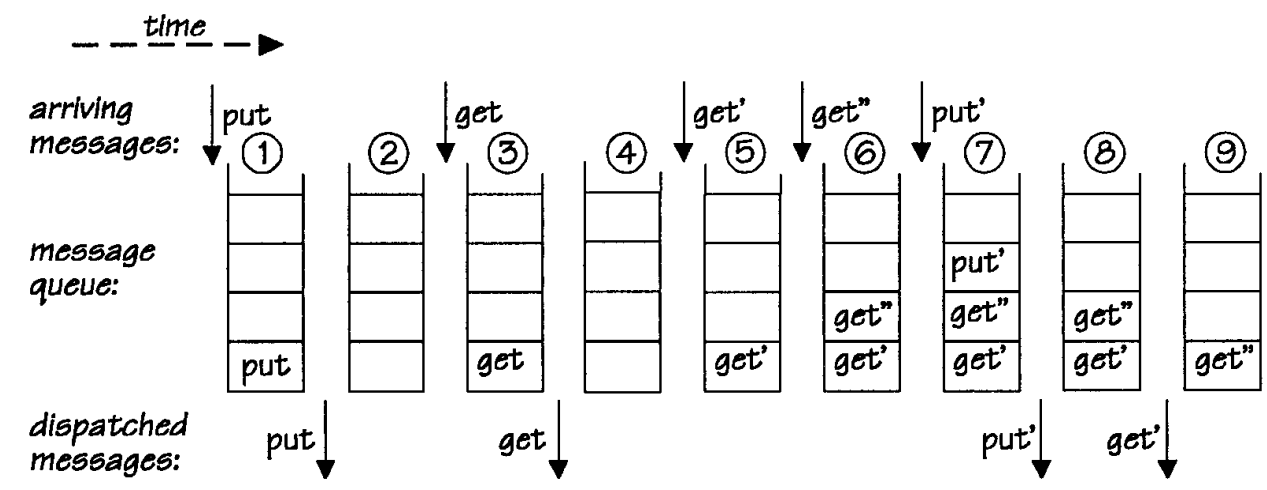

FIG. 15. Example of message scheduling in a bounded buffer. 


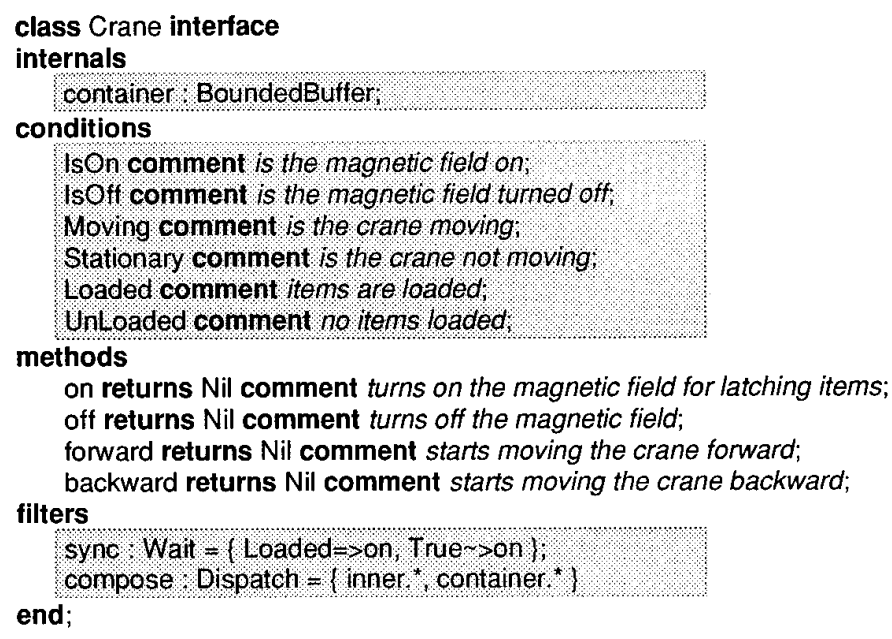

FIG. 16. Sina definition of the interface of class Crane.

part "True $>$ on" has the following meaning: all the received messages can match this expression except the message with the selector $o n$. If the received message has the selector on and the condition Loaded is false, then it will be blocked until the condition Loaded becomes true. If the message matches the wait filter, then it will proceed to the dispatch filter. Otherwise, the message will be blocked until it can be accepted by the Wait filter.

The dispatch filter has two parts and does not define any conditions. The first part "inner." has the following meaning. If the selector of the received message corresponds to one of the methods of class Crane, then the message will be dispatched to that method. If not, then the message will be evaluated with respect to the second part "container.*". Note that container is an internal object and an instance of class BoundedBuffer. If the selector of the received message corresponds to one of the methods of class BoundedBuffer, then it will be forwarded to container. Before dispatching to one of its methods, the message has to pass through the filters of container as well. This means that the received message has to fulfill the synchronization conditions of both classes Crane and BoundedBuffer.

This example illustrates how composition-filters can be used to compose synchronization specifications defined by different classes. Notice that the dispatch filter of class Crane implements an inheritance mechanism since the methods of class BoundedBuffer are now available at the interface of class Crane. This mechanism is also known as composition-based inheritance.

\subsubsection{Definition of Class ProtectedCrane Using Filters}

Class ProtectedCrane inherits from class Crane and introduces a new synchronization constraint which restricts the execution of the methods on and off. Since the method on is also restricted by class Crane, the synchronization constraints of Crane and ProtectedCrane must be composed together for this method. The interface definition of class ProtectedCrane is shown in Fig. 17. To implement composition-based inheritance, class ProtectedCrane declares myCrane of class Crane as an internal object. Further, ProtectedCrane inherits all the conditions declared by Crane by using the expression "myCrane.*" in the conditions clause.

Class ProtectedCrane declares two filters. The first filter protect is an instance of Wait and is used to enforce the synchronization constraint of ProtectedCrane. If a message is received by an instance of class ProtectedCrane, it will be first evaluated with respect to the filter part "Stationary $=>$ on, off $\}$ ", which has the following meaning: if the received message has one of the selectors on or off and if the condition Stationary evaluates to true then this part matches the message. If the received message does not match, then it will be evaluated with respect to the second part "True $>$ \{on, off $\}$ ". This part accepts all the messages except the messages with the selectors on and off. Therefore, the on and off messages can pass this wait filter only if the condition Stationary is true. Otherwise, they will be blocked until the condition Stationary changes to true.

The dispatch filter inherit has only one part "myCrane.*". A message that arrives at the dispatch filter is forwarded to the instance myCrane of class Crane if the selector of the message corresponds to one of the interface methods of class Crane. Before dispatching to one of these methods, the message has to pass through the filters of classes Crane and BoundedBuffer, and therefore has to

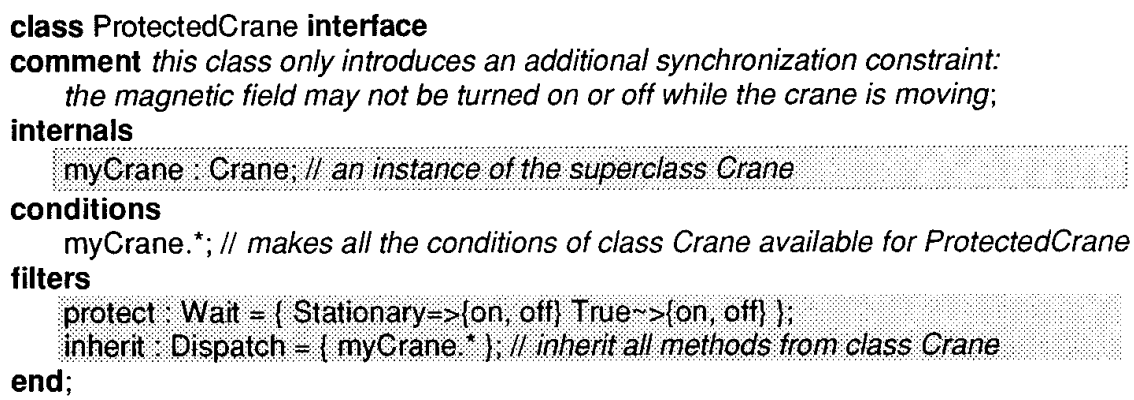




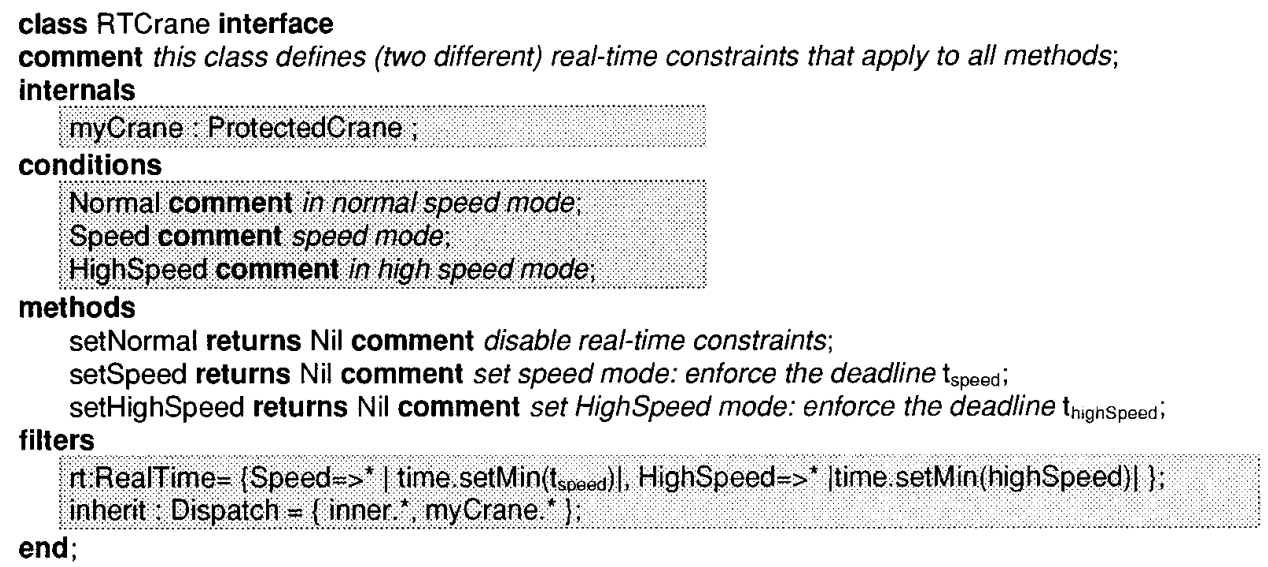

FIG. 18. Sina definition of the interface of class RTCrane.

satisfy all the synchronization constraints of these classes as well. ${ }^{9}$

\subsection{Composing Real-Time Constraints Using Filters}

\subsubsection{Definition of Class RTCrane Using Filters}

Class RTCrane inherits from class ProtectedCrane and depending on the mode of its instance object, it introduces two real-time constraints. The interface definition of class RTCrane is defined as depicted in Fig. 18. Class RTCrane declares an internal object myCrane of class ProtectedCrane. The three conditions Normal, Speed and HighSpeed are declared following the conditions clause.

Class RTCrane declares two filters, $r t$ and inherit, which are instances of classes RealTime and Dispatch, respectively. The composition-filters model assumes that timing information travels with each message. The real-time filter is used to affect the timing attribute of message if the corresponding message matches the filter. If the message does not match the filter, then it will pass to the next filter without being affected by the filter.

The real-time filter of class RTCrane has two parts. The first part is specified as "Speed $=>^{*}\left|\operatorname{time} \cdot \operatorname{setMin}\left(t_{\text {speed }}\right)\right|$ ". Here, if the condition Speed is true, then this part matches all the possible messages. If the message matches this part, then it will receive the timing constraint expressed between the separators "| ... |". If the message does not match the first part, then it will be evaluated with respect to the second part of the real-time filter. The condition Speed is set to true by invoking the method setSpeed. In addition, this method assigns false to the other conditions Normal and HighSpeed.

The real-time constraint in the first part of the filter is expressed as $\mid$ time.minEnd $\left(t_{\text {speed }}\right) \mid$. Here, time denotes

\footnotetext{
${ }^{9}$ In certain cases, it it necessary to enforce synchronization constraints by wait filters atomically at a single class. To specify atomicity, a filter from a "superclass" can be inserted, e.g., by declaring "myCrane.sync" in the filter set of class ProtectedCrane. In our case example, however, the atomic enforcement of synchronization constraints was not found necessary.
}

the timing attribute of the received message. The method minEnd is defined by time to associate a timing constraint with it. The method minEnd accepts a time specification as a value and assigns it as the deadline of the execution. However, this is only done if the new value is smaller than the previous value (the minimum is taken). If the condition Speed evaluates to true, then the real-time filter of class RTCrane associates with every message the timing value $t_{\text {Speed. }}$ if $t_{\text {Speed }}$ is smaller that the current timing value of the received message.

The second part of the real-time filter is declared as "HighSpeed $=>* \mid$ time.setMin(HighSpeed) $\mid$ ". If the condition HighSpeed evaluates to true, then every message is associated with the timing value $t_{\text {Highspeed }}$, provided that this value is smaller that the current timing value of the received message. The condition HighSpeed is set to true by invoking the method setHighSpeed. The invocation of this method will make the other conditions false.

If the method setNormal is invoked, then the conditions Speed and HighSpeed are set to false. As a result, the received message cannot match the real-time filter and therefore will be forwarded to the dispatch filter without modifying the timing attribute.

The dispatch filter makes the methods of class ProtectedCrane available at the interface, provided that the received message can pass through the filters of class ProtectedCrane. If, for example, the condition HighSpeed is true, the received message has the selector off and the crane is moving, then this message will pass through the real-time and dispatch filters, but will be blocked by the wait filter of class ProtectedCrane. This means that in this particular implementation, the synchronization constraints have a higher priority than the real-time constraints. Since class RTCrane introduces soft timing constraints, we consider this priority assignment preferable.

\subsubsection{Definition of Class EmergencyRTCrane Using Filters}

Class EmergencyRTCrane inherits from RTCrane and introduces a hard timing constraint for the method emer- 


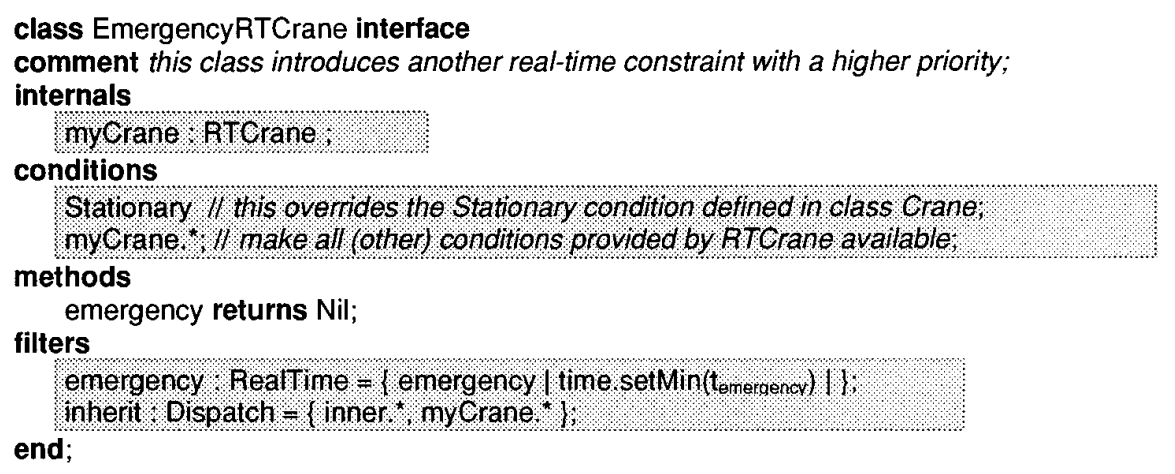

FIG. 19. Sina definition of the interface of class EmergencyRTCrane.

gency. If invoked, the method emergency must be executed within its time limit independent of other real-time and synchronization constraints. Consider the interface definition of class EmergencyRTCrane that is depicted in Fig. 19. To implement composition-based inheritance, class EmergencyRTCrane declares myCrane as an instance of class $R T C r a n e$ following the internals clause. Further, class EmergencyRTCrane declares the condition Stationary and "inherits" all the conditions defined by RTCrane using the expression "myCrane.*". Note that the condition Stationary was declared before by class ProtectedCrane. Redefinition of a condition overrides the previous definition. The purpose of this redefinition will be explained in the following paragraphs.

Class EmergencyRTCrane defines two filters: a real-time filter and a dispatch filter. The real-time filter has only one part. If the received message has the selector emergency, then it will receive the real-time deadline $t_{\text {emergency }}$ provided that $t_{\text {emergency }}$ is smaller than the current deadline. All other requests will be passed to the dispatch filter without being affected by this real-time filter. The dispatch filter either dispatches the message to the method emergency or to the internal object myCrane of class RTCrane. The implementation of class EmergencyRTCrane is shown in Fig. 20.

The method emergency carries out two kinds of actions. First, it turns off the magnetic field by invoking the method off on server. In the Sina language, server denotes the receiver of the message and it is comparable to the pseudo- variable self in Smalltalk or this in $\mathrm{C}++$. Further, the Sina language provides the pseudo-variable client which denotes the sender of a message. After shutting off the magnetic field, the method emergency generates warning signals.

Now assume that the received message has the selector emergency and has no timing constraints. The instance of class EmergencyRTCrane will then assign the timing attribute $t_{\text {emergency }}$ to the message and forward it to the dispatch filter. The dispatch filter will execute the method emergency. The method emergency will then invoke the method off on server, which is the same instance that received the emergency request. This self invocation will be forwarded by the dispatch filter to the internal object myCrane of class RTCrane. Assume that the crane has been put in HighSpeed mode previously. Then the realtime filter of RTCrane will try to assign the timing attribute $t_{\text {Highspeed }}$ to the message. However, since $t_{\text {emergency }}$ is smaller than $t_{\text {HighSpeed, }} t_{\text {emergency }}$ will be kept as the timing attribute of the message. The internal object myCrane of class RTCrane will further forward the message to its own internal object which is an instance of class ProtectedCrane. If the crane is moving, then the wait filter of class ProtectedCrane will normally block the off and on requests, because its condition Stationary will be false. This would give a higher priority to the synchronization constraints, which is not desired for class EmergencyRTCrane.

To give a higher priority to the real-time constraint of the method emergency than the synchronization constraints of

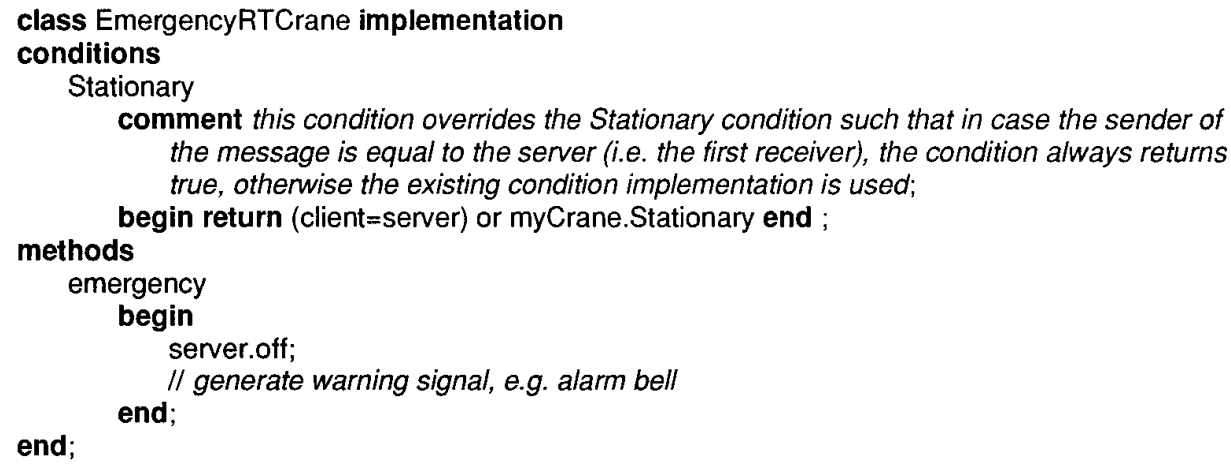

FIG. 20. Sina definition of the implementation of class EmergencyRTCrane. 
class ProtectedCrane, the condition Stationary is redefined by class EmergencyRTCrane. The condition Stationary evaluates to true if the message is a self call (client=server), or the condition Stationary evaluates to true by the internal object myCrane (myCrane.Stationary). Polymorphically redefining conditions allows programmers to give different priorities to constraints along the inheritance hierarchy.

\section{RELATED WORK}

In the following, we will present the related work first in concurrent, and later in real-time object-oriented languages.

\subsection{Concurrent Object-Oriented Languages}

In this section, we will give a list of significant examples of concurrent object-oriented languages. In the following subsections, we will evaluate these languages using the three requirements presented in Section 3.3.

We observe four distinct approaches in object-oriented synchronization schemes. These are namely, explicit acceptance, activation conditions, meta-level, and interobject level synchronizations. Explicit acceptance means that during a method execution, the state of the object is considered, and based on this it is explicitly decided what message to accept next. For example, ABCL/1 [55], POOL [10], and Eiffel// [18] support this approach.

Activation conditions define for each message whether it should be accepted or blocked. In some languages the activation conditions can be manipulated directly (e.g., Actor languages [1] and Hybrid [42]). In other languages and systems the activation conditions are specified indirectly, e.g., through guards (e.g., in [22], Guide [20], and Concurrency Annotations [33]), path expressions (e.g., PROCOL [14]), or state abstractions (e.g., ACT ++ [31], Rosette [53], and Synchronizing Actions [41]). The proposal in [38] combines activation conditions through guards with state abstractions.

Concurrent reflective languages define synchronization constraints at a meta-level. Examples of this are ABCL/ R [54], Actalk [16], and MAUD [23].

Another approach toward specifying synchronization is at the interobject level; synchronization problems can be solved by controlling the patterns of interactions between objects. Although such approaches generally do not suffer from inheritance anomaly, they inflict with modularity and the notion of autonomous objects, thereby causing other problems in reusability and extensibility. Examples of interobject level synchronization specifications are atomic transactions [40], atomic delegations [4], Abstract Communication Types [7], and Synchronization Patterns [34].

\subsubsection{Definition of the Synchronization Inheritance Anomaly Problem}

During the past several years, various researchers $[10,15,30,43]$ have indicated that there is an interference between inheritance and concurrency. The identification and description of inheritance anomalies was first made by Matsuoka, Yonezawa and Wakita in [35, 37]. Basically, this work classified the inheritance anomalies with respect to representation and manipulation of object states. Here, the anomalies were termed "mixing synchronization specification with application code," "state-partitioning," "orthogonally restricting specifications," "history- sensitiveness," etc.

Our definition of anomalies is based on the generic synchronization model presented in section 3.2. Our analysis is somewhat more general. For example, In Section 3.3.1, we generalized the "mixing synchronization specification with application code" anomaly to the problem of "nonmodular synchronization specifications." We claimed that the modularity definition must not only include the separation of synchronization specification from application code, but also that the synchronization specification must consist of an independent State Abstraction and Condition Mapping Functions, as depicted in Fig. 7. In fact our generic model made it possible to compare the synchronizationrelated inheritance problems to the problems of inheriting real-time constraints.

\subsubsection{Evaluation of Languages with Respect to Synchronization Inheritance Anomalies}

Modularity of Synchronization Specifications. In a number of concurrent object-oriented languages with explicit message acceptance, each object defines a part called the body; this is a piece of code that is executed independently from the execution of incoming messages (e.g. POOL-I [11], Eiffel// [18]). In these systems, the body represents the high-level specification of a process that is associated with the object: the received messages are considered and when deemed appropriate, the execution of corresponding methods is initiated. It is obvious that the body approach requires complete redefinition of the body in a subclass whenever the synchronization changes. In particular, in the subclass all the synchronization contraints for the methods defined in parent classes must be defined again. This conflicts with incremental specification. It also violates encapsulation, because synchronization constraints that are implementation-dependent must be redefined as well. This creates a dependency between the subclass and the superclasses: redefining the synchronization of the superclass requires (all) the subclasses to update the body implementations.

In addition to realizing the synchronization constraints of the object, the body may implement some general house keeping operations as well. Modifying these operations (i.e., application code) cannot be done independently from synchronization, thus requiring additional unwanted redefinitions.

Composability of Synchronization Constraints. The requirement for polymorphic synchronization specifications states that a certain synchronization constraint must be 
applicable to multiple methods, either in the same class or in subclasses. The languages that adopt guard-like approaches such as Guide [20], Concurrency Annotations [33] and Synchronisers [38 $]^{10}$ cannot effectively specify polymorphic synchronization specifications.

In some languages the objective of synchronization synthesis has been discarded, usually motivated by the complexity of the problem and the relatively small amount of reused code. Examples of this are POOL-I [11], Eiffel// [18], and Dragoon $[12,48]$. In these languages synchronization specifications can be inherited (once), but not extended afterwards.

Expressiveness for State Abstractions. The expressive power for specifying state abstractions varies widely among synchronization schemes. At one end of the scale are mechanisms such as path expressions, which can only express acceptance based on very specific information (i.e., the history of executed messages). Extended path expressions provide guards to extend the expressive power. Another specific mechanism for expressing synchronization is based on synchronization counters [49]. The more sophisticated synchronization schemes (e.g., in [22, 38]) allowalmost-the full expressiveness of the programming language for expressing synchronization conditions.

A solution to the bookkeeping problem of maintaining history information as defined in Section 3.3.3 is available in some systems through the definition of generic pre- and post-actions that are executed for all messages that are received by the object (e.g., in Encapsulators [47], or in reflective languages such as ABCL/R [36] and MAUD [23]).

It should be noted that the approaches based on behavioral abstraction that perform a become statement at, or after the end of methods (e.g., ACT ++ [31]) are obliged to predict the state of the object at the moment of message arrival. Thus they cannot deal with any information about arrived messages in the time between the become and the (test for) acceptance of a particular message.

\subsection{Real-Time Object-Oriented Programming Languages}

In the following paragraphs, we will present some significant real-time object-oriented languages. The evaluation of these languages will be presented in the next section.

The Maruti programming language (MPL) [44] is based on the $\mathrm{C}++$ language and designed for the Maruti distributed operating system. MPL provides a number of constructs to specify timing constraints. These constructs can be applied to message sends and code blocks only. Timing constraints of the client object are passed on ${ }^{11}$ to the server

\footnotetext{
${ }^{10}$ Although this is less severe because their approach supports behavioral abstractions as well, which can cope with this particular problem.

${ }^{11}$ In real-time languages, sometimes the term inheritance is used for this purpose as well. In this paper, we only use the term inheritance when we refer to class inheritance.
}

object and to the methods called by it. MPL offers several synchronization constructs that are orthogonal to the object structure and are embedded in the method bodies of an object.

$\mathrm{RTC}++[27]$ is an extension of the $\mathrm{C}++$ language and is suitable for programming both soft and hard real-time applications. RTC ++ is implemented on top of the realtime distributed operating system kernel arts. Timing constraints can be associated either with method headers or declared in the method body. Thus timing constraints may be visible in the method interface and be encapsulated in the object's implementation. RTC ++ offers a guard-like synchronization mechanism, declared at the interface of every method.

The FLEX language [32] is based on the $\mathrm{C}++$ language and associates real-time constraints with code blocks. Any change in the execution state causes only those immediately dependent constraints to be checked, thus no propagation of the constraints is done. The constraints may be labeled and thus referred to by other constraints. Precedence relations can be specified using constraint blocks that refer to attributes of other constraints. Constraints may also contain boolean expressions which are treated as an assertion to be maintained throughout the block's lifetime. Synchronization must be specified with the constraints embedded in method implementations as well.

RealTimeTalk [17] is based on the Smalltalk language without the features of Smalltalk that impede the timing prediction, such as method lookup and garbage collection. RealTimeTalk has been targeted to provide frameworks for soft and hard real-time applications. In the framework, objects of a special class Use-Case encapsulate timing-critical tasks. The RealTimeTalk compiler generates C-code which, in turn, has to be compiled to a target system. RealTimeTalk cannot specify explicit synchronization constraints.

The DROL language [52] is based on the $\mathrm{C}++$ language and aims at programming distributed realtime systems. Its eminent feature is that users can describe the semantics of message communications at a meta-level as a communication protocol by using sending and receiving primitives. Like RTC ++, DROL is implemented on top of the artskernel. The concept behind DROL is to provide the best effort at the server, taking into account the timing constraints o a message, and to realize the least suffering at the client side by detecting timing errors and avoiding the propagation thereof. Timing constraints can be specified for both messages and methods. For each method of a (server) object, its worst-case execution time may be specified. Each message send may be a so-called time polymorphic invocation. The server chooses from a set of alternatives the method that meets the timing constraint. Periodic actions are specified using the active keyword, followed by the method declaration and parameters specifying the time bound of period and execution time. Because of the time polymorphic invocations, DROL provides flexible computations and graceful degradation. In DROL, timing con- 
straints for message sends may only be declared in method bodies. Timing constraints for message acceptance specify the worst case time and may only be declared at the object interface. Synchronization in DROL is based on the enabled-set approach [53].

\subsubsection{Definition of the Real-Time Inheritance Problem}

To the best of our knowledge, apart from our related work [8], real-time constraints inheritance anomalies have not been studied in the literature. Our definition in [8] is somewhat different from the analysis that is presented in this paper. In [8], real-time constraint inheritance anomalies were classified as "mixing real-time specifications with application code," "nonpolymorphic real-time specifications" and "orthogonally restricting real-time specifications." The "mixing real-time specifications with application code' anomaly is a special case of the modularity anomaly described in Section 4.2.1. The "nonpolymorphic" and "orthogonally restricting" real-time specification anomalies correspond to respectively the polymorphic real-time specifications and real-time synthesis anomalies described in Section 4.3.2. The analysis technique presented in this paper is based on the generic real-time model and is more general. In addition, the generic model made it possible to analyze synchronization and real-time inheritance anomalies together.

\subsubsection{Evaluation of Languages with Respect to Real- Time Inheritance Anomalies}

We will now evaluate the real-time object-oriented languages with respect to the real-time synchronization constraint inheritance anomalies.

The Maruti programming language is only capable of expressing timing and synchronization constraints within methods; timing and synchronization constraints are always encapsulated and cannot be separately inherited nor reused in subclasses. Therefore, MPL suffers from both the modularity and the composability inheritance anomalies in both the synchronization and the real-time domain.

In RTC ++ timing constraints can be declared either at the method interface or in the method body. If the latter is the case, this will cause the modularity anomaly. Otherwise, the timing (and synchronization) constraints are defined per method header. As a result, the composability anomaly applies as well. Expressing guards for synchronization of RTC ++ has several limitations, resulting in the expressiveness anomaly.

Flex associates both synchronization and timing constraints with statements, or blocks of statements. Therefore the modularity requirement is not met, resulting in anomalies. Although blocks can be labeled for access by other constraints, the constraints themselves cannot be reused by other constraints, resulting in composability anomalies.

Because RealTimeTalk does not allow for subclassing of classes with real-time specifications, and also does not support explicit synchronization constraints, the notion of inheritance anomaly does not apply to RealTimeTalk.

DROL offers two distinct timing specification approaches. Timing constraints for message sends are embedded in the method body of the sender, causing modularity and composability inheritance anomalies. The (worst case) timing for message reception is declared at the interface of the object, and cannot be reused separately, causing composability anomalies. Time polymorphic invocations are powerful, but do not contribute directly to the composability of real-time constraints. The synchronization approach suffers from modularity anomaly, since the enabled sets are determined inside the method body.

\section{EVALUATION}

In Section 5.2, the requirements for effectively composing and reusing synchronization and real-time constraint specifications were summarized by three points: separation of concerns, expression power and assigning priorities of specifications. We will now evaluate the composition-filters approach with respect to these requirements.

\subsection{Separation of Concerns}

Separation of concerns is one of the essential requirements to compose and reuse synchronization and real-time constraint specifications. Within this context, the following four requirements were identified:

1. Separation of synchronization and real-time specifications and method executions.

2. Separation of the elements of synchronization specifications.

3. Separation of the elements of real-time specifications.

4. Composition of concerns.

\subsubsection{Synchronization Specification, Real-Time Specification, and Method Execution}

The composition-filters approach separates the concerns of specifying synchronization and real-time constraints and inheriting and executing methods by three different filters which are functionally independent of each other. Each filter affects the received messages in a certain way. For example, wait filters may delay messages, real-time filters may affect the timing characteristic of messages and dispatch filters may execute the corresponding methods. Specification of filters are separated from the implementation of objects.

\subsubsection{Elements of Synchronization Specifications}

In Section 3.2, the elements of our generic synchronization model were defined as Implicit State, Synchronization Conditions, Message Acceptance, Scheduler and Methods. The mapping functions State Abstraction, Synchronization Condition Mapping, and Method Executions relate these 
elements to each other. We will now analyze how elements of synchronization specifications are modeled within the composition-filters approach.

Consider, for example the specification of the wait filter of class Crane: $\{$ Loaded $=>$ on, True $\sim>$ on $\}$. Here, the condition Loaded corresponds to the element Synchronization Condition. The element State Abstraction Function is defined by the implementation of the condition method Loaded. The operator "=>" corresponds to Synchronization Condition Mapping Function. The " > " operator specifies an exclusive Condition Mapping Function. An exclusive Condition Mapping Function provides an openended specification of synchronization conditions. For example, the wait filter of class Crane only restricts the messages with the selector on. Message Acceptance is specified as a set. In the wait filter specification of class Crane, "on" refers only to the method on of class Crane. A filter specification may refer to the total message inteface of a class. For example, in the dispatch filter of Crane, the specification "container.*" refers to the total message interface of class BoundedBuffer. The character "*" provides an open-ended specification of object's behavior which may be extended by new compositions.

Synchronization Condition, Synchronization Condition Mapping and Message Acceptance can be combined in various ways. For example, the specification "Loaded $=>$ on" is a one-to-one combination. The specification "True $>$ on" is a one-to-many combination. ${ }^{12}$

\subsubsection{Elements of Real-Time Specifications}

In real-time filters, most elements of real-time specifications are similar to synchronization specifications. The evaluation presented in the previous section is also valid for real-time filters. In addition, real-time filters express real-time constraints delimited by the character "|". Realtime constraints can be associated with a set of methods and conditions. For example, the specification "Speed $=>$ " * |time.setMin $\left(t_{\text {Speed }}\right) \mid$ " relates the real-time constraint $t_{\text {Speed }}$ to all the messages received by the object and to the condition Speed.

\subsubsection{Composition of Concerns}

The composition-filters model allows compositions of separately specified constraints. For example, Class ProtectedCrane, as defined in Section 6.2.1, composes the synchronization constraint of class Crane and class ProtectedCrane for the method on. This is the so-called AND composition of constraints. A received message has to pass through two different filters, thus satisfying the conditions

\footnotetext{
${ }^{12}$ It is also possible to specify many-to-many combinations, for example by using the expression " $S(\#)=>\#$.*". This expression can be interpreted as follows: All the interface methods of the objects that satisfy the condition $S$. This expression is very useful in specifying the so-called Associative Inheritance mechanism and is explained in [5]. For brevity, we do not discuss the many-to-many combinations in this paper.
}

of both filters. An example for or composition of constraints is illustrated by classes BoundedBuffer and Crane. These classes enforce synchronization constraints on different sets of methods. The or composition in class Crane is defined through the usage of multiple parts in a filter. The operator ",", in fact, is a Conditional-or composition operator. Using different filters provides separation of concerns of different application-domain requirements, such as concurrent and real-time systems.

\subsection{Expression Power}

As we outlined before in the discussion about the inheritance anomalies, sufficient expression power for specifying the mapping functions is relevant for two reasons: firstly to be able to express certain aspects, such as deciding message acceptance based on the number of active threads in the object. Secondly, in some cases such a requirement can be expressed through extra code in the implementation of the object. As we have shown previously, such an approach will generally lead to inheritance anomalies. Implicit Object State covers a virtually unlimited state-space. Therefore, the most obvious area where expressive power may be lacking is in the State Abstraction Function. As the composition filters approach offers the full functionality of method implementations for expressing state abstraction mapping, most results can be defined in a straightforward manner. For example in the guards approach of Guide [20], the guard expressions are restricted to a logical expression over instance variables and synchronization counters. In addition, condition methods can be inherited or polymorphically redefined. For example, classes ProtectedCrane and EmergencyRTCrane inherit the condition methods of their superclasses. Class EmergencyRTCrane polymorphically redefines the condition of class ProtectedCrane. We should note that the redefinition of conditions may have unwanted consequences, as improper usage may violate the synchronization constraints from the superclass. On the otherhand, the equivalent mechanism of polymorphic method overriding is widely accepted in object-oriented programming.

The expression power of real-time filters is equivalent to the expression power of most real-time languages. A detailed description of real-time specifications in the composition-filters model is given in [8].

\subsection{Assign Priorities to Specifications}

In the composition-filters model, a message may have to pass through the filters of several objects, before being dispatched to the corresponding method. Consider, for example, class $R T C r a n e$ which receives a message with the selector on. This message has to pass through the filters of RTCrane, ProtectedCrane, and Crane before being dispatched to the method on. Real-time filters may affect the timing attribute of messages, whereas wait filters may block them. This means that in case of AND composition, the 
synchronization specification will prevail the real-time specification. This was illustrated in the definition of class RTCrane. As in the definition of class EmergencyRTCrane, to give a higher priority to real-time specifications, the synchronization conditions may be polymorphically redefined.

\subsection{Conclusion}

Recently, a considerable number of concurrent and realtime object-oriented languages have been introduced. However, composing and reusing object-oriented programs with both synchronization and real-time constraints has not been addressed, even though most real-world systems are concurrent and have some real-time aspects. In short, failing in providing composable synchronization and real-time constraints results in so-called synchronization and real-time inheritance anomalies. This implies that the reuse and composition of real-time and concurrent objects is unnecessarily limited.

To analyze these inheritance anomalies, we presented generic object-oriented models for synchronization and real-time constraint specifications. These models are more general than the previously proposed ones, and led us to identify a set of requirements that are necessary to avoid the inheritance anomaly problem.

To satisfy the identified requirements we extended the object-oriented model modularly with composition filters. These modular and orthogonal extensions allow us to overcome both synchronization and real-time constraint inheritance anomalies. A number of examples using the Sina language were shown to illustrate the applicability of the proposed mechanism. For a more precise description of composition-filters and the Sina language, we refer to $[13,29]$.

\section{REFERENCES}

1. G. Agha, An overview of actor languages. ACM SIGPLAN Notices 21, 10 (Oct. 1986), 58-67.

2. M. Akşit and A. Tripathi, Data abstraction mechanisms in Sina/ST. Proc. of the OOPSLA ' 88 Conference. ACM SIGPLAN Notices 23, 11 (Nov. 1988), 265-275.

3. M. Akșit, On the design of the object-oriented language Sina. Ph.D. thesis, Univ. of Twente, 1989.

4. M. Akşit, J. W. Dijkstra, and A. Tripathi, Atomic delegation: Objectoriented transactions. IEEE Software 8, 2 (Mar. 1991), 84-92.

5. M. Akşit, L. Bergmans, and S. Vural, An object-oriented languagedatabase integration model: The composition-filters approach. Proc. of the ECOOP' 92 Conference. Lecture Notes in Computer Science, Vol. 615. Springer-Verlag, Berlin/New York, 1992, pp. 372-395.

6. M. Akssit and L. Bergmans, Obstacles in object-oriented software development. Proc. of the OOPSLA'92 Conference. ACM SIGPLAN Notices 27, 10 (Oct. 1992), 341-358.

7. M. Akşit, K. Wakita, J. Bosch, L. Bergmans, and A. Yonezawa, Abstracting object-interactions using composition-filters. In ObjectBased Distributed Processing (R. Guerraoui, O. Nierstrasz, and M. Riveill, Eds.). Lecture Notes in Computer Science, Vol. 791. SpringerVerlag, Berlin/New York, 1993, pp. 152-184.
8. M. Akşit, J. Bosch, W. v.d. Sterren, and L. Bergmans, Real-time specification inheritance anomalies and real-time filters. Proc of the ECOOP '94 Conference. Lecture Notes in Computer Science, Vol. 821. Springer Verlag, Berlin/New York, July 1994, pp. 386-407.

9. J. Allen, Maintaining knowledge about temporal intervals. Comm. ACM 26, 110, (1983), 832-843.

10. P. America, POOL-T: A parallel object-oriented language. In ObjectOriented Concurrent Programming (A. Yonezawa and M. Tokoro, Eds.). The MIT Press, Cambridge, MA, 1987, pp. 199-220.

11. P. America, A parallel object-oriented language with inheritance and subtyping. Proc. of the OOPSLA/ECOOP '90 Conference. ACM SIGPLAN Notices 25, 10 (Oct. 1990), 161-168.

12. C. Atkinson, S. Goldsack, A. Di Maio, and R. Bayan, Object oriented concurrency and distribution in DRAGOON. J. Object-Oriented Programming March/April (1991), 11-18.

13. L. Bergmans, Composing concurrent objects. Ph.D. thesis, Univ. of Twente, 1994.

14. J. van den Bosch and C. Laffra, PROCOL-A parallel object language with protocols. Proc. of the OOPSLA '89 Conference. ACM SIGPLAN Notices 24, 10 (1989), 95-102.

15. J-P. Briot and A. Yonezawa, Inheritance and synchronization in concurrent OOP. Proc of the ECOOP'87 Conference. Springer-Verlag, Berlin/New York, 1987, pp. 32-40.

16. J.-P. Briot, Actalk: A testbed for classifying and designing Actor languages in the Smalltalk-80 environment. Proc. of the ECOOP ' 89 Conference, Cambridge Univ. Press, Nottingham, July 10-14, 1989, pp. 109-129.

17. E. Brorsson, C. Eriksson, and J. Gustafsson, RealTimeTalk: An object-oriented language for hard real-time systems. Proc. of IFAC International Workshop on Real-Time Systems, Brugge, 1991.

18. D. Caromel, Concurrency: An object-oriented approach. TOOLS-2 (J. Bezivin, B. Meyer, and J. M. Nerson, Eds.). 1990, pp. 183-197.

19. B. Dasarathy, Timing constraints of real-time systems: Constructs for expressing them, methods for validating them. IEEE Trans. Software Eng. 11, 1 (1985), 80-86.

20. D. Decouchant, P. Le Dot, M. Riveill, C. Roisin, and X. Rousset de Pina, A synchronization mechanism for an object-oriented distributed system. Proc. of the 11th IEEE Conference on Distributed Computing, May 1991.

21. W. van Dijk and J. Mordhorst, CFIST, composition filters in Smalltalk. Graduation report, HIO Enschede, May 1995.

22. S. Frølund, Inheritance of synchronization constraints in concurrent object-oriented programming languages. Proc. of the ECOOP' 92 Conference. Lecture Notes in Computer Science, Vol. 615. SpringerVerlag, Berlin/New York, 1992, pp. 185-196.

23. S. Frølund and G. Agha, A language framework for multi-object coordination. Proceedings of the ECOOP '93 Conference. Lecture Notes in Computer Science, Vol. 707, Springer-Verlag, Kaiserlautern, Germany, July 1993, pp. 346-360.

24. E. Gamma, R. Helm, R. Johnson, and J. Vlissides, Design Patterns: Elements of Reusable Object-Oriented Software. Addison-Wesley, Reading, MA, 1995.

25. M. Glandrup, Extending $\mathrm{C}++$ using the concepts of composition filters. M.Sc. thesis, University of Twente, Nov. 1995.

26. A. Goldberg and D. Robson, Smalltalk-80: The Language and Its Implementation. Addison-Wesley, Reading, MA, 1983.

27. Y. Ishikawa, H. Tokuda, and C. W. Clifford, Object-Oriented RealTime Language Design. Carnegie-Mellon Univ. 1990.

28. I. Jacobson, M. Christerson, P. Jonsson, and G. Overgaard, ObjectOriented Software Engineering-A Use Case Driven Approach. Addison-Wesley/ACM Press, Reading, MA/New York, 1992.

29. P. Koopmans, On the definition and implementation of the Sina/St language. M.Sc. thesis, Univ. of Twente, July 1995. 
30. D. G. Kafura and K. H. Lee, Inheritance in Actor based concurrent object-oriented languages. Proceedings of ECOOP '89. Cambridge Univ. Press, Nottingham, July 10-14, 1989, pp. 131-145.

31. D. G. Kafura and K. H. Lee, ACT ++ : Building a concurrent $\mathrm{C}++$ with Actors. J. Object-Oriented Programming 3, 1 (May/June 1990), pp. $25-37$.

32. K. J. Lin, J. W. S. Liu, K. B. Kenny, and S. Natarajan, Flex: A language for programming flexible real-time Systems. In Foundations of Real-Time Computing: Formal Specifications and Methods. (A. M. van Tilborg and G. M. Koob, Eds.). Kluwer, Dordrecht, 1991, pp. 251-290.

33. K.-P. Löhr, Concurrency annotations. Proc. of the OOPSLA '92 Conference. ACM SIGPLAN Notices 27, 10 (Oct. 1992), 327-340.

34. C. V. Lopes and K. Lieberherr, Abstracting process-to-function relations in concurrent object-oriented applications. Proc. of the ECOOP '94 Conference. Lecture Notes in Computer Science, Vol. 821. Springer Verlag, July 1994, pp. 81-99. Berlin/New York.

35. S. Matsuoka, K. Wakita, and A. Yonezawa, Synchronization constraints with inheritance: What is not possible-So what is? Internal report, Tokyo Univ., 1990.

36. S. Matsuoka, T. Watanabe, and A. Yonezawa, Hybrid group reflective architecture for object-oriented concurrent reflective programming. Proc. of the ECOOP' 91 Conference. Lecture Notes in Computer Science, Vol. 512. Springer-Verlag, Berlin/New York, 1991. pp. 213-250.

37. S. Matsuoka and A. Yonezawa, Inheritance anomaly in object-oriented concurrent programming languages. In Research Directions in Concurrent Object-Oriented Programming (G. Agha, P. Wegner, and A. Yonezawa, Eds.). MIT Press, Cambridge, MA, Apr. 1993, pp. 107-150.

38. S. Matsuoka, K. Taura, and A. Yonezawa, Highly efficient and encapsulated re-use of synchronization code in concurrent object-oriented languages. Proc. of the OOPSLA '93 Conference. ACM SIGPLAN Notices 28, 10 (Oct. 1993), 109-126.

39. C. McHale, Synchronization in concurrent object-oriented languages: Expressive power, genericity and inheritance. Ph.D. thesis, Trinity College, Univ. of Dublin. 1994.

40. J. E. B. Moss, Nested Transactions: An Approach to Reliable Computing. MIT Press, Cambridge, MA, 1985.

41. C. Neusius, Adapting synchronization counters to the requirements of inheritance. ACM SIGPLAN OOPS Messenger 2, 4 (Oct. 1991).

42. O. M. Nierstrasz, Active objects in Hybrid. Poc. of the OOPSLA '87 Conference. ACM SIGPLAN Notices 22, 12 (Dec. 1987), 243-253.

43. O. Nierstrasz, The next 700 concurrent object-oriented languagesReflections on the future of object-based concurrency. In Object Composition. Centre Universitaire d'Informatique, Univ. of Geneva, June 1991, pp. 165-187.

44. V. M. Nirkhe, S. K. Tripathi, and A. K. Agrawal, Language support for the Maruti real-time system. Proc. 1990 Real-Time Systems Symposium. IEEE Comput. Soc. Press, Los Alamitos, CA, 1990, pp. 257-266.

45. Proc. of the OOPSLA'94 Conference. ACM SIGPLAN Notices 29, 10 (Oct. 1994).

46. Proc. of the OOPSLA'95 Conference. ACM SIGPLAN Notices 30, 10 (Oct. 1995).

47. G. A. Pascoe, Encapsulators: A new software paradigm in Smalltalk-

Received November 1, 1995; revised February 1, 1996; accepted April 25, 1996
80. Proc. of the OOPSLA '86 Conference. ACM SIGPLAN Notices 21, 11 (Nov. 1986), 341-346.

48. S. C. Reghizzi, G. G. de Paratesi, and S. Genolini, Definition of reusable concurrent software components. Proc. of the ECOOP'91 Conference. Lecture Notes in Computer Science, Vol. 512. SpringerVerlag, Berlin/New York, 1991, pp. 148-166.

49. P. Robert and J.-P. Verjus, Toward autonomous descriptions of synchronization modules. Information Processing 77, North-Holland, Amsterdam, 1977, pp. 981-986.

50. J. Rumbaugh, M. Blaha, W. Premerlani, F. Eddy, and W. Lorensen, Object-Oriented Modeling and Design. Prentice-Hall, Englewood Cliffs, NJ, 1991.

51. B. Stroustrup, The $C++$ Programming Language. Addison-Wesley, Reading, MA, 1986.

52. K. Takashio and M. Tokoro, DROL: An object-oriented programming language for distributed real-time systems. Proc of the OOPSLA '92 Conference. ACM SIGPLAN Notices 27, 10 (Oct. 1992) 276-294.

53. C. Tomlinson and V. Singh, Inheritance and synchronization with enabled-sets. Proc. of the OOPSLA '89 Conference. ACM SIGPLAN Notices 24, 10 (Oct. 1989), 103-112.

54. T. Watanabe and A. Yonezawa, Reflection in an object-oriented concurrent language. Proc. of the OOPSLA '88 Conference. ACM SIGPLAN Notices 23, 11 (Nov. 1988), 306-315.

55. A. Yonezawa, E. Shibayama, T. Takada, and Y. Honda, Modeling and programming in an object-oriented concurrent language ABCL/ 1. In Object-Oriented Concurrent Programming (A. Yonezawa and M. Tokoro, Eds.). The MIT Press, Cambridge, MA, 1987, pp. 55-89.

LODEWIJK BERGMANS studied computer science in The Netherlands on the Faculty of Computer Science, University of Twente. He received a Ph.D. from the University of Twente. His dissertation, entitled "Composing Concurrent Objects," deals with object-oriented concurrent systems, addressing the issues of reusable concurrency and synchronization in object-oriented programming languages and analysis and design methods, including graphical notations and implementation aspects. Currently, he is active as an independent consultant on object-oriented software engineering for STEX bv, The Netherlands. He is also associated with the TRESE Project at the University of Twente. Dr. Bergmans' main research interests include the various aspects of object-oriented software engineering, in particular extensibility and reusability of concurrent objects, analysis and design methods, project management, software architectures, and application frameworks.

MEHMET AKȘIT holds an M.Sc. from the Technical University of Eindhoven and a Ph.D. from the University of Twente. Currently, he is working as an associate professor at the University of Twente. He is the leader of the Twente Research and Education on Software Engineering (TRESE) Project. Dr. Akşit has been the user and developer of objectoriented systems since 1983. He specializes in the application of objectoriented methods to practical software systems. He is the principal inventor of object-composition-filters, atomic delegations, associative inheritance and delegation mechanisms, abstract communication types, and fuzzy-logic-based object-oriented methods and metrics. He has identified and defined a set of new problems related to object-oriented analysis and design. His areas of interest are object-oriented analysis and design of industrial software systems, object-oriented CASE tools, and programming environments, languages, and methods. 